\title{
ROOT GROWTH: HOMOGENIZATION IN DOMAINS WITH TIME DEPENDENT PARTIAL PERFORATIONS
}

\author{
Yves CAPdeboscQ $^{1}$ And Mariya PtashnyK ${ }^{2}$
}

\begin{abstract}
In this article we derive a macroscopic model for the time evolution of root density, starting from a discrete mesh of roots, using homogenization techniques. In the microscopic model each root grows vertically according to an ordinary differential equation. The roots growth rates depend on the spatial distribution of nutrient in the soil, which also evolves in time, leading to a fully coupled non-linear problem. We derive an effective partial differential equation for the root tip surface and for the nutrient density.
\end{abstract}

Mathematics Subject Classification. 35B27, 35K55, 92C99.

Received October 28, 2010. Revised June 27, 2011.

Published online September 14, 2011.

\section{Introduction}

In this work, we consider the growth of a system of parallel and densely distributed cylindrical roots. The roots growth direction is dictated by gravity: fixed and vertical. The rate of growth of each root depends on the the nutrient concentration $c$ in the surrounding soil. The time evolution of the length $\rho$ of each root depends on the length of the root, its horizontal position and on $c$. It is modelled by a differential equation

$$
\frac{\mathrm{d} \rho}{\mathrm{d} t}=r(t, x, \rho, c)
$$

see Section 2 for the precise form of the function $r$. The nutrient concentration is affected (decreased) by the roots growth. Away from the roots, the evolution of the nutrient concentration in the soil is given by a conservation law,

$$
\partial_{t} c-\nabla_{x} \cdot\left(D(x) \nabla_{x} c\right)=0 \text { in the soil, }
$$

where the function $D$ determines the diffusion of the nutrient in the soil. The nutrient consumption of each plant happens on the surface of its root,

$$
D \nabla c \cdot \nu=-g(c) \text { on any root surface, }
$$

where, at each point $y$ on a root surface, $\nu(y)$ is the outward normal, and $g$ is a non-negative function. Precise assumptions on $D$ and $g$ are detailed in Section 2 .

\footnotetext{
Keywords and phrases. Homogenization, root growth, time dependent domains.

1 Mathematical Institute, 24-29 St Giles', Oxford OX1 3LB, UK. capdeboscq@maths.ox.ac.uk

2 Department of Mathematics I, RWTH Aachen University, Wüllnerstr. 5b, 52056 Aachen, Germany.

ptashnyk@math1.rwth-aachen.de
} 


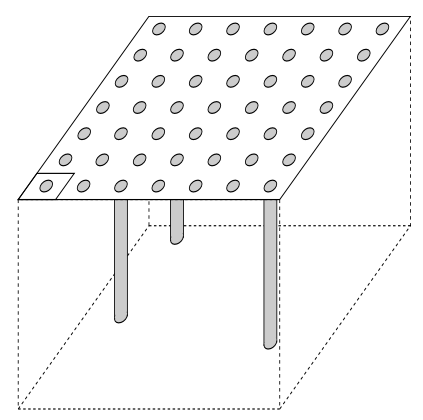

FIGURE 1. Sketch of a configuration at time $t$. The roots grow vertically, and are planted following a rectangular lattice. They are of variable length. The nutrient $c$ is located in the three dimensional domain below the surface, except on the locus of the roots.

The goal of this work is to derive a macroscopic model of root growth, in presence of many roots. We assume that the roots are planted periodically in a square patch, with a periodicity cell $\varepsilon Z$ where $Z=$ $\left\{\left(y_{1}, y_{2}\right) \in[0,1] \times[0,1]\right\}$. Each root has a cross-section $\varepsilon S$, where

$$
S=\left\{\left(y_{1}, y_{2}\right) \text { s.t. }\left|\left(y_{1}, y_{2}\right)-(1 / 2,1 / 2)\right|<\alpha\right\}
$$

with $\alpha<1 / 2$. We derive an effective model for the root density in the soil and for the nutrient concentration when $\varepsilon$ tends to zero.

The motivation for this work is plant physiology. The plant anchorage, water and nutrient uptake depend on the root growth and on the root architecture. Thus understanding root growth is of particular interest to plant physiologists, and different models have been developed. Some models are centred on the topological description of the complicated network of root system, Other focus on the growth of individual roots from which such networks would occur. In early models the change of length of a single root (or of a root mass) was modelled using ordinary differential equation [5,11], namely $\mathrm{d} l / \mathrm{d} t=v f(l)$, for a given growth rate $v$.

The growth of a single root branch can also be modelled by a combination of discrete and continuous ansatz [6]. In such a case, the growth (in length) of each single cell in a root branch is described by an ordinary differential equation. A discrete cell concentration model then provides a model for the growth of a whole root branch.

The general principle of root architecture models is to define a dynamic topological network of organs at various stages using morphogenetic models, and to simulate the growth of these organs using concepts such as sources and sinks, $[9,12,15,17-19]$. The morphogenetic rules are defined using the Lindenmayer algorithm (L-system). Interactions between modules (root branches) and environment can be captured by generalisations of L-systems. Some root architecture models allow the growth direction to depend on the gradient of nutrient concentration in the soil (or medium) [7,20]. Models of root architecture based on root branching density were considered in [9].

For a very dense root network, representative for roots growing in a flooding soil or in medium, one can consider the root density distribution and define a continuous model for the growth of a root network [4]. This model is a system consisting of a transport equation for the root tip density $n$, and an ordinary differential equation for root length density $\rho$,

$$
\partial_{t} n+\nabla \cdot(\mathbf{v} n)=f(\rho), \quad \partial_{t} \rho=|\mathbf{v}| n,
$$

where $\mathbf{v}$ is a growth velocity vector. The growth velocity depends on the root density and on the nutrient concentration in the medium or in the soil.

The model we study here does not attempt to address the difficult issue of roots inter-twinning. Our focus is to rigorously connect a discrete root model to a continuous one, taking into account the influence of the environment. We make an important simplifying assumption, namely that the roots grow vertically. In this 
simplified framework, the main mathematical difficulty is that the three-dimensional domain in which the nutrient is defined, that is, the soil below the square patch outside of the roots, depends non-linearly on time and on $\varepsilon$.

This paper is structured as follows. The precise description of the model we consider is given in Section 2 . In Section 3, we introduce our main results. We show in Proposition 3.1 that the microscopic model introduced in Section 2 is well-posed. The effective macroscopic problem obtained in the limit $\varepsilon \rightarrow 0$ is given by Theorem 3.2 . The rest of the paper is devoted to the proof of these results. Section 4 is devoted to the proof of Proposition 3.1, whereas Section 5 is devoted to the proof of Theorem 3.2. We conclude this paper by remarks on possible extensions of this work.

\section{Problem Formulation}

In this section, we detail the mathematical model we consider. We refer to $\Omega$ as the cubic domain $\Omega=(0,1)^{3}$. A point in $\Omega$ is denoted $x=\left(x_{1}, x_{2}, x_{3}\right)$, we use the notation that $x^{\prime}=\left(x_{1}, x_{2}\right)$. When referring to microscopic variables, we write $y=\left(y_{1}, y_{2}, y_{3}\right)$, and $y^{\prime}=\left(y_{1}, y_{2}\right)$. On the microscopic level, we denote $Z=(0,1)^{2}$ the two dimensional unit cell and the cross section of a root is $S=\left\{\left|y^{\prime}-(1 / 2,1 / 2)\right|<\alpha\right\}$ where $0<\alpha<1 / 2$. The part of the unit cell not occupied by the root is $Y=Z \backslash \bar{S}$.

We denote by $\varepsilon=1 / N$ the small parameter. It is the ratio between the minimum distance between the centre of base of two roots and the size of the domain $\Omega$, and $N$ is a positive integer. We note $N(\varepsilon)=\varepsilon^{-2}$, the number of roots. The parameter $i$ denotes a multi-index, $i \in\{1, \ldots, N(\varepsilon)\}=\left\{\left(i_{1}, i_{2}\right) \in\{0, \ldots, N-1\}^{2}\right\}$. The base of the $i$-th root is

$$
S_{i}^{\varepsilon}=\varepsilon(S+i)=\left\{x=\left(x^{\prime}, 0\right) \text { with }\left(x^{\prime} / \varepsilon-i\right) \in S\right\},
$$

and, at time $t \geq 0$ the $i$-th root is $R_{t, i}^{\varepsilon}$, given by

$$
R_{t, i}^{\varepsilon}=S_{i}^{\varepsilon} \times(0, \rho(i \varepsilon, t))
$$

and we note the set of all roots at time $t$ by $R_{t}^{\varepsilon}=\cup_{i=0}^{N(\varepsilon)} R_{t, i}^{\varepsilon}$. The immersed boundary of $R_{t, i}^{\varepsilon}$ is $\Gamma_{t}^{\varepsilon}=\cup_{i=0}^{N(\varepsilon)} \partial R_{t, i}^{\varepsilon} \backslash$ $\left(S_{i}^{\varepsilon} \times\{0\}\right)$.

The time-dependent domain where the evolution equation for the nutrient $c$ is defined is

$$
\Omega_{t}^{\varepsilon}=\Omega \backslash R_{t}^{\varepsilon}
$$

The initial root length distribution $\rho^{0}$ is regular and given at $N(\varepsilon)$ grid points $i \varepsilon$ by $\rho^{0}(i \varepsilon)$. The initial $i$-th root is $R_{0, i}^{\varepsilon}=S_{i}^{\varepsilon} \times\left(0, \rho_{0}(i \varepsilon)\right)$, and the initial set of roots is $R_{0}^{\varepsilon}=\cup_{i=0}^{N(\varepsilon)} R_{0, i}^{\varepsilon}$. The initial domain is $\Omega_{0}^{\varepsilon}=\Omega \backslash R_{0}^{\varepsilon}$, and the initial immersed root boundary is $\Gamma_{0}^{\varepsilon}=\cup_{i=0}^{N(\varepsilon)} \partial R_{0, i}^{\varepsilon} \backslash\left(S_{i}^{\varepsilon} \times 0\right)$.

In the time dependent domain $\Omega_{t}^{\varepsilon}$, the concentration $c^{\varepsilon}$ is a solution of the following parabolic problem

$$
\begin{array}{ll}
\partial_{t} c^{\varepsilon}-\nabla \cdot\left(D^{\varepsilon} \nabla c^{\varepsilon}\right)=0 & \text { in } \Omega_{t}^{\varepsilon}, t \in(0, T), \\
D \nabla c^{\varepsilon} \cdot \nu=-\varepsilon \lambda c^{\varepsilon} & \text { on } \Gamma_{t}^{\varepsilon}, t \in(0, T), \\
D \nabla c^{\varepsilon} \cdot \nu=0 & \text { on } \partial \Omega_{t}^{\varepsilon} \backslash \Gamma_{t}^{\varepsilon}, t \in(0, T), \\
c^{\varepsilon}=c^{0} & \text { in } \Omega_{0}^{\varepsilon},
\end{array}
$$

where $\lambda>0$ represents the rate of absorption of nutrient by the root axis, and where $D$ is the diffusion coefficient. We assume that this coefficient is smooth, $D \in C^{1}(\bar{\Omega})$, and non-degenerate for all time: there exists a two positive constants $0<d_{0} \leq d_{1}<\infty$ such that

$$
0<d_{0}<D \leq d_{1} \text { for all } x \in \bar{\Omega} \text {. }
$$


The initial density of nutrient is smooth, that is,

$$
c^{0} \in C^{1}(\bar{\Omega}) \text { and } c^{0} \geq 0
$$

We also assume that the initial distribution of root lengths is regular, namely

$$
\rho^{0} \in C^{1}\left([0,1]^{2}\right) \text { and } 0<\rho^{0} \leq K
$$

The evolution of $\rho_{i}^{\varepsilon}$ is defined by the differential equation

$$
\begin{gathered}
\frac{\mathrm{d}}{\mathrm{d} t} \rho_{i}^{\varepsilon}=\frac{c s(t, i, \varepsilon)}{\sqrt{1+c s^{2}(t, i, \varepsilon)}}\left(K-\rho_{i}^{\varepsilon}\right), \\
\rho_{i}^{\varepsilon}(0)=\rho^{0}\left(\varepsilon x_{i}^{\prime}\right),
\end{gathered}
$$

where $K<1$ is the maximum possible root length, where the growth velocity $c s(t, i, \varepsilon)$ depends on time, and on the amount of nutrient available. This dynamic models the fact that the roots have a maximal size $K$, a maximal growth rate (equal to 1) and grow faster if more nutrient is available. The specific choice $c s / \sqrt{1+c s^{2}}$ is arbitrary. We suppose in this work that the quantity $c s$ is given by

$$
c s(t, i, \varepsilon)=\int_{\Omega_{t}^{\varepsilon} \cap B\left(\left(i \varepsilon, \rho_{i}^{\varepsilon}\right), r_{0}\right)} c^{\varepsilon}(t, x) \mathrm{d} x,
$$

where $r_{0}$ is a fixed positive constant. The definition (2.3) means that $c s$, and in turn the growth rate, depends on the total concentration of nutrient within a distance $r_{0}$ of the tip of the root. This root growth model is inspired by $[10,16]$. It is related to the model studied in [4], where the growth velocity depends on the nutrient concentration with a saturation effect: the roots can only take up to a maximal nutrient concentration. Note that this implies

$$
0 \leq \frac{\mathrm{d}}{\mathrm{d} t} \rho_{i}^{\varepsilon} \leq K, \text { and } \rho^{0}\left(\varepsilon x_{i}^{\prime}\right) \leq \rho_{i}^{\varepsilon} \leq K \text { for all } i \text { and } t
$$

\section{Main Results}

The goal of this paper is to derive a macroscopic model corresponding to the microscopic model (2.1)-(2.3) when $\varepsilon$ tends to zero, i.e. for a very dense root structure. In Section 4, we show that the microscopic model (2.1)-(2.3) is well-posed, in the sense, that the solution exists, is unique, and is controlled by the initial condition.

Proposition 3.1. For every $\varepsilon>0$ there exists a unique weak solution $c^{\varepsilon}$ of (2.1) and $\rho^{\varepsilon}$ of (2.2). The concentration $c^{\varepsilon}$ satisfies the estimate

$$
\left\|c^{\varepsilon}\right\|_{L^{\infty}\left(0, T ; L^{\infty}\left(\Omega_{t}^{\varepsilon}\right)\right)}+\left\|\partial_{t} c^{\varepsilon}\right\|_{L^{2}\left(0, T ; L^{2}\left(\Omega_{t}^{\varepsilon}\right)\right)}+\left\|\nabla c^{\varepsilon}\right\|_{L^{\infty}\left(0, T ; L^{2}\left(\Omega_{t}^{\varepsilon}\right)\right)}+\varepsilon^{1 / 2}\left\|c^{\varepsilon}\right\|_{L^{\infty}\left((0, T) ; L^{2}\left(\Gamma_{t}^{\varepsilon}\right)\right)} \leq \mu,
$$

where $\mu$ depends on $\left\|c^{0}\right\|_{H^{1}\left(\Omega_{0}^{\varepsilon}\right)},\left\|c^{0}\right\|_{L^{\infty}\left(\Omega_{0}^{\varepsilon}\right)}$, $\left\|\rho^{0}\right\|_{C^{1}\left((0,1)^{2}\right)}$ and $K$ only. Furthermore, $c^{\varepsilon}$ is non negative.

In fact, in the course of the proof of Proposition 3.1, we verify that the solution of (2.1) can be computed as a limit of an iterative procedure involving only the resolution of linear elliptic systems, see Section 4.3.

Section 5 is devoted to the rigorous derivation of the limit macroscopic model. The main result of this paper is the following. 
Theorem 3.2. The solutions of the microscopic model (2.1)-(2.3) converge to the solution $\rho \in W^{1, \infty}\left(0, T ; W^{1, \infty}\right.$ $\left.\left((0,1)^{2}\right)\right), c \in L^{2}\left(0, T ; H^{1}(\Omega)\right) \cap H^{1}\left(0, T ; L^{2}(\Omega)\right)$ of the system

$$
\begin{aligned}
v^{*} \partial_{t} c-\operatorname{div}\left(D^{*} \nabla c\right)+R^{*} c & =0 \quad \text { in } \Omega \times(0, T), \\
D^{*} \nabla c \cdot \nu & =0 \quad \text { on } \partial \Omega \times(0, T), \\
c(t=0, x) & =c^{0} \quad \text { in } \Omega .
\end{aligned}
$$

The functions $v^{*}$ and $R^{*}$ are given by

$$
v^{*}(t, x)=\left\{\begin{array}{ll}
|Y| & \text { when } x_{3}<\rho\left(t, x^{\prime}\right), \\
1 & \text { otherwise },
\end{array} \text { and } R^{*}(t, x)= \begin{cases}\lambda|\partial S| & \text { when } x_{3}<\rho\left(t, x^{\prime}\right), \\
0 & \text { otherwise. }\end{cases}\right.
$$

The matrix-valued function $D^{*}$ is diagonal, with entries

$$
D_{11}^{*}(t, x)=D_{22}^{*}(t, x)= \begin{cases}D(x)\left(d^{*}+|Y|\right) & \text { when } x_{3}<\rho\left(t, x^{\prime}\right), \\ D(x) & \text { otherwise }\end{cases}
$$

and $D_{33}^{*}(t, x)=D(x)$. The constant $d^{*}$ is a given by

$$
d^{*}=\int_{Y} \partial_{y_{1}} \omega_{1} \mathrm{~d} y
$$

where the corrector function $\omega_{1} \in H^{1}(Y)$ is the $Z$-periodic solution of

$$
-\Delta \omega_{1}=0 \text { in } Y, \quad-\nabla\left(\omega_{1}-y_{1}\right) \cdot \nu=0 \quad \text { on } \partial S, \quad \int_{Y} \omega_{1} \mathrm{~d} y=0 .
$$

The effective root length $\rho$ is the solution of the differential equation

$$
\begin{aligned}
\partial_{t} \rho & =\frac{c s\left(t, x^{\prime}\right)}{\sqrt{1+c s^{2}\left(t, x^{\prime}\right)}}(K-\rho) \quad \text { in }(0, T) \times(0,1)^{2} \\
\rho\left(t=0, x^{\prime}\right) & =\rho^{0}\left(x^{\prime}\right) \quad \text { in }(0,1)^{2},
\end{aligned}
$$

where the growth rate depends on the concentration c by means of the relation

$$
c s\left(t, x^{\prime}\right)=\int_{\Omega \cap B\left(\left(x^{\prime}, \rho\left(t, x^{\prime}\right)\right), r_{0}\right)} c(t, \zeta) \mathrm{d} \zeta .
$$

Remark. Note that in the effective nutrient concentration model, the space dependence of the coefficients is now solely due to $\rho$, which determines the surface $x_{3}=\rho\left(t, x^{\prime}\right)$ which is the boundary between two regimes for the coefficients $v^{*}, D^{*}$ and $R^{*}$. The differential equation satisfied by $\rho$ is unchanged, in terms of its dependence on $c s$. But since $c s$ depends itself on $\rho$ and $c$, the dynamic is in fact modified. This effective model for $c$ has a reaction term, $\lambda|\partial S| c$, where roots are present. This accounts for the uptake of nutrients by the roots. The macroscopic velocity of the moving boundary between the domain with roots and the domain without roots reflect the non-local feature of the microscopic growth velocity of a single root. As expected, this macroscopic model is simpler than the microscopic one, but it retains a strong dependence on the initial root distribution and nutrient density. It is easy to see that if $D, c^{0}$ and $\rho^{0}$ are constant, the root boundary will be flat. In other situations, the surface $x_{3}=\rho\left(t, x^{\prime}\right)$ will be more complex. 


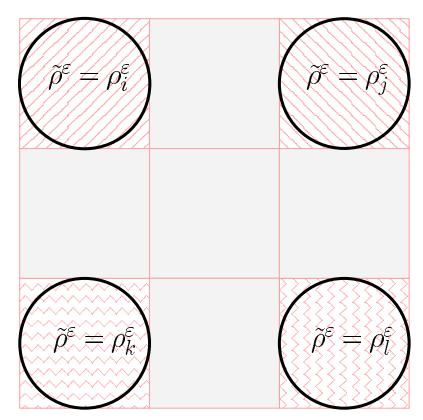

FIGURE 2. A piecewise linear interpolation of the root lengths, where $\tilde{\rho}^{\varepsilon}$ is constant on the squares limiting the disk cross-sections of the roots, and piecewise linear and continuous elsewhere.

\section{WELL-POSEDNESS OF THE MODEL PROBLEM}

The goal of this section is to prove that the model given by (2.1)-(2.3) is well posed, that is, that there exists a unique solution to the problem, and furthermore that the solutions $c^{\varepsilon}$ and $\rho^{\varepsilon}$ are controlled by the initial data in appropriate norms, independently of $\varepsilon$. Our approach to this problem will be to study the properties of the solutions, assuming they exist, then prove existence using a compactness argument, and finally prove uniqueness using the regularity of the weak solutions. The main difficulty here is the time dependence of the domain. The dependence of the domain on the small parameter $\varepsilon$ is classical in homogenization.

We start by mapping the problem to a time-independent domain.

\subsection{Transformation to the time-independent domain $\Omega_{0}^{\varepsilon}$}

Because the domain on which the problem is posed is time dependent, we cannot directly apply general results on the existence and regularity of $c^{\varepsilon}$ and $\rho^{\varepsilon}$. We therefore construct a change of variable mapping $\Omega_{t}^{\varepsilon}$ to a fixed domain $\Omega_{0}^{\varepsilon}$.

For $0<L_{0}<L_{1}<1$, and $0 \leq z \leq 1$, consider the function $\varphi\left(L_{0}, L_{1}, z\right)=\tilde{\varphi}\left(L_{0}, z / L_{1}\right)$ such that $z \rightarrow$ $\varphi\left(L_{0}, L_{1}, z\right) \in C^{2}([0,1])$ maps $[0,1]$ onto $[0,1]$, satisfies $\varphi\left(L_{0}, L_{1}, L_{1}\right)=L_{0}$ and $\varphi\left(L_{0}, L_{1}, 1\right)=1$. It is given by given by

$$
\begin{aligned}
\tilde{\varphi}\left(L_{0}, z\right) & =z L_{0} \text { for } z \in[0,1] \\
& =z L_{0}+(z-1)^{3} \frac{L_{1}^{2}\left(L_{1}-L_{0}\right)}{\left(1-L_{1}\right)^{3}} \text { for } z \in\left[1, L_{1}^{-1}\right] .
\end{aligned}
$$

Note that $\partial_{3} \varphi, \partial_{23} \varphi$ and $\partial_{33} \varphi$ are continuous functions on $[0, K] \times(0,1) \times[0,1]$.

Choose a continuous, piecewise $C^{1}$ function $\tilde{\rho}^{\varepsilon}$ matching exactly the root lengths on each root, that is,

$$
\rho^{0} \leq \tilde{\rho}^{\varepsilon}<K, \quad \text { and } \tilde{\rho}^{\varepsilon}\left(t, x^{\prime}\right)=\rho_{i}^{\varepsilon}(t) \text { for all } i \in\left\{1, \ldots, N_{\varepsilon}\right\} \text { and } x^{\prime} \in S_{i}^{\varepsilon}, t>0 .
$$

Note that the upper bound $K$ is consistent with the other constraints, as $K$ is the upper asymptotic limit of $\rho_{i}^{\varepsilon}$ for all $i$. Specifically, choose $\tilde{\rho}^{\varepsilon}$ to be a piecewise linear interpolation between the root lengths (as done in Fig. 2 for example).

Note that this spatial interpolation is linear in each $\rho_{i}^{\varepsilon}$, and is time independent. Therefore, $\partial_{t} \tilde{\rho}^{\varepsilon}$ is the same spatial interpolation between the time derivatives $\partial_{t} \rho_{i}^{\varepsilon}$. In particular, it takes its values in $(0, K)$.

The spatial variation of $\tilde{\rho}^{\varepsilon}$ depends on $\varepsilon$. To quantify this dependence, we introduce

$$
V\left(\rho^{\varepsilon}\right)\left(t, x^{\prime}\right)=\max \left\{\frac{\left|\rho_{i}^{\varepsilon}(t)-\rho_{j}^{\varepsilon}(t)\right|}{\varepsilon}, \text { over all } i, j \text { s.t. }|i-j| \leq 2,\left|x^{\prime} / \varepsilon-i\right| \leq 1\right\} .
$$


Because $\tilde{\rho}^{\varepsilon}$ is a linear interpolant we see that for some constant $C>0$ independent of $x^{\prime}$ and $\varepsilon$, and $t$,

$$
C V\left(\rho^{\varepsilon}\right)\left(t, x^{\prime}\right) \leq\left|\nabla_{x^{\prime}} \tilde{\rho}^{\varepsilon}\left(t, x^{\prime}\right)\right| \leq \frac{1}{C} V\left(\rho^{\varepsilon}\right)\left(t, x^{\prime}\right) \text { for all } x^{\prime} \in(0,1)^{2}, t>0
$$

Note that $V\left(\rho^{\varepsilon}\right)$ depends on $\varepsilon$. Similarly, we have, with obvious notations,

$$
C V\left(\partial_{t} \rho^{\varepsilon}\right)\left(t, x^{\prime}\right) \leq\left|\nabla_{x^{\prime}} \partial_{t} \tilde{\rho}^{\varepsilon}\left(t, x^{\prime}\right)\right| \leq \frac{1}{C} V\left(\partial_{t} \rho^{\varepsilon}\right)\left(t, x^{\prime}\right) \text { for all } x^{\prime} \in(0,1)^{2}, t>0
$$

The map $\mathcal{F}_{\varepsilon}:\left(t, x_{1}, x_{2}, x_{3}\right) \rightarrow\left(t, x_{1}, x_{2}, \varphi\left(\rho^{0}, \tilde{\rho}^{\varepsilon}, x_{3}\right)\right)$ is a change of variable between $(0, T) \times \Omega_{t}^{\varepsilon}$ and $(0, T) \times \Omega_{0}^{\varepsilon}$. The a priori bounds on $\rho^{\varepsilon}$ and $\varphi$ allow to prove the following proposition.

Proposition 4.1. The transformation $\mathcal{F}_{\varepsilon}$ from $(0, T) \times \Omega_{t}^{\varepsilon}$ onto $(0, T) \times \Omega_{0}^{\varepsilon}$ satisfies

$$
\frac{1}{K} \min _{(0,1)^{2}} \rho^{0} \leq \mathcal{J}\left(\tilde{\rho}^{\varepsilon}\right) \leq 1+\frac{3}{1-K} \max _{(0,1)^{2}}\left(\frac{K}{\rho^{0}}-1\right),
$$

where $\mathcal{J}\left(\tilde{\rho}^{\varepsilon}\right)$ is the Jacobian determinant of the transformation given by

$$
\mathcal{J}\left(\tilde{\rho}^{\varepsilon}\right)=\left|\operatorname{det}\left(D \mathcal{F}_{\varepsilon}\right)\right|=\partial_{3} \varphi\left(\rho^{0}, \tilde{\rho}^{\varepsilon}, x_{3}\right)
$$

Proof. We have

$$
\partial_{3} \varphi\left(\rho^{0}, \tilde{\rho}^{\varepsilon}, x_{3}\right)=\frac{1}{\tilde{\rho}^{\varepsilon}} \partial_{2} \tilde{\varphi}\left(\rho^{0}, \frac{x_{3}}{\tilde{\rho}^{\varepsilon}}\right) .
$$

For $z \leq 1, \partial_{2} \tilde{\varphi}\left(\rho^{0}, z\right)=\rho^{0}$. For $1 \leq z \leq 1 / \tilde{\rho}^{\varepsilon}, \partial_{2} \tilde{\varphi}\left(\rho^{0}, z\right)$ is non decreasing, since $\tilde{\rho}^{\varepsilon} \geq \rho^{0}$, with

$$
\tilde{\varphi}\left(\rho^{0}, \frac{1}{\tilde{\rho}^{\varepsilon}}\right)=\rho^{0}+3 \frac{\tilde{\rho}^{\varepsilon}-\rho^{0}}{1-\tilde{\rho}^{\varepsilon}} \leq \rho^{0}+3 \frac{K-\rho^{0}}{1-K},
$$

since $\tilde{\rho}^{\varepsilon} \leq K$. Therefore,

$$
\frac{\rho^{0}}{K} \leq \partial_{3} \varphi\left(\rho^{0}, \tilde{\rho}^{\varepsilon}, x_{3}\right) \leq 1+\frac{3}{\rho^{0}} \frac{K-\rho^{0}}{1-K}
$$

since $\rho^{0} \leq \tilde{\rho}^{\varepsilon} \leq K$.

We now proceed with change of variables in equations (2.1). Introducing the notation

$$
w=f\left(\tilde{\rho}^{\varepsilon}, t, x^{\prime}, x_{3}\right)=\varphi\left(\rho^{0}, \tilde{\rho}^{\varepsilon}, x_{3}\right),
$$

using the monotonicity and continuity of $\varphi$ with respect to $x_{3}$ and we obtain for $\tilde{c}^{\varepsilon}\left(t, x^{\prime}, w\right)=c^{\varepsilon}\left(t, x^{\prime}, f^{-1}\left(\tilde{\rho}^{\varepsilon}\right.\right.$, $\left.\left.t, x^{\prime}, w\right)\right)$ with the notation

$$
\tilde{c}^{\varepsilon}\left(t, x^{\prime}, f\left(\tilde{\rho}^{\varepsilon}, t, x^{\prime}, x_{3}\right)\right)=c^{\varepsilon}\left(t, x^{\prime}, x_{3}\right),
$$

the following equations defined in the time independent domain $\Omega_{0}^{\varepsilon}$,

$$
\begin{array}{ll}
v\left(\tilde{\rho}^{\varepsilon}\right) \partial_{t} \tilde{c}^{\varepsilon}+b\left(\tilde{\rho}^{\varepsilon}\right) \partial_{w} \tilde{c}^{\varepsilon}-\nabla \cdot\left(M\left(\tilde{\rho}^{\varepsilon}\right) \nabla \tilde{c}^{\varepsilon}\right)=0 & \text { in }(0, T) \times \Omega_{0}^{\varepsilon}, \\
M\left(\tilde{\rho}^{\varepsilon}\right) \nabla \tilde{c}^{\varepsilon} \cdot \nu=-\varepsilon v\left(\tilde{\rho}^{\varepsilon}\right) \lambda \tilde{c}^{\varepsilon} & \text { on }(0, T) \times \Gamma_{0}^{\varepsilon}, \\
M\left(\tilde{\rho}^{\varepsilon}\right) \nabla \tilde{c}^{\varepsilon} \cdot \nu=0 & \text { on }(0, T) \times\left(\partial \Omega_{0}^{\varepsilon} \backslash \Gamma_{0}^{\varepsilon}\right), \\
\tilde{c}^{\varepsilon}=c^{0}\left(x^{\prime}, w\right) & \text { in } \Omega_{0}^{\varepsilon}, \text { at } t=0,
\end{array}
$$


where $v\left(\tilde{\rho}^{\varepsilon}\right)=1 / \mathcal{J}\left(\tilde{\rho}^{\varepsilon}\right), b\left(\tilde{\rho}^{\varepsilon}\right)=\partial_{t} f\left(\tilde{\rho}^{\varepsilon}, \cdot\right) / \mathcal{J}\left(\tilde{\rho}^{\varepsilon}\right)$, and the matrix $M\left(\tilde{\rho}^{\varepsilon}\right)$ is given by

$$
M\left(\tilde{\rho}^{\varepsilon}\right)=\frac{D^{\varepsilon}}{\mathcal{J}\left(\tilde{\rho}^{\varepsilon}\right)}\left(\begin{array}{ccl}
1 & 0 & \partial_{x^{\prime}} f\left(\tilde{\rho}^{\varepsilon}, \cdot\right) \\
0 & 1 & \\
\partial_{x^{\prime}} f\left(\tilde{\rho}^{\varepsilon}, \cdot\right)^{T} & \left|\partial_{x^{\prime}} f\left(\tilde{\rho}^{\varepsilon}, \cdot\right)\right|^{2}+\left|\mathcal{J}\left(\tilde{\rho}^{\varepsilon}\right)\right|^{2}
\end{array}\right),
$$

where $D^{\varepsilon}\left(x^{\prime}, f\left(\tilde{\rho}^{\varepsilon}\right)\right)=D\left(x^{\prime}, x_{3}\right)$. Remark that at $t=0$, the map $\mathcal{F}_{\varepsilon}$ is the identity map, therefore the initial condition is preserved.

When no ambiguity exists on $\tilde{\rho}^{\varepsilon}$, that is, everywhere but in Section 4.3 , we will use the simpler notations

$$
v^{\varepsilon}=v\left(\tilde{\rho}^{\varepsilon}\right), b^{\varepsilon}=b\left(\tilde{\rho}^{\varepsilon}\right), f^{\varepsilon}=f\left(\tilde{\rho}^{\varepsilon}, \cdot\right), \mathcal{J}^{\varepsilon}=\mathcal{J}\left(\tilde{\rho}^{\varepsilon}\right) \text {, and } M^{\varepsilon}=M\left(\tilde{\rho}^{\varepsilon}\right) .
$$

\subsection{A priori estimates}

For the sake of clarity, we recall the definition of a solution to (4.4).

Definition 4.2. The function $\tilde{c}^{\varepsilon}$ is a weak solution of (4.4) if $\tilde{c}^{\varepsilon} \in L^{2}\left(0, T ; H^{1}\left(\Omega_{0}^{\varepsilon}\right)\right), \partial_{t} \tilde{c}^{\varepsilon} \in L^{2}\left(0, T ; L^{2}\left(\Omega_{0}^{\varepsilon}\right)\right)$ such that

$$
\int_{0}^{\tau} \int_{\Omega_{0}^{\varepsilon}}\left(v^{\varepsilon} \partial_{t} \tilde{c}^{\varepsilon} \phi+b^{\varepsilon} \partial_{w} \tilde{c}^{\varepsilon} \phi+M^{\varepsilon} \nabla \tilde{c}^{\varepsilon} \nabla \phi\right) \mathrm{d} x^{\prime} \mathrm{d} w \mathrm{~d} t=-\varepsilon \int_{0}^{\tau} \int_{\Gamma_{0}^{\varepsilon}} \lambda v^{\varepsilon} \tilde{c}^{\varepsilon} \phi \mathrm{d} \gamma^{\varepsilon} \mathrm{d} t
$$

for all $\phi \in L^{2}\left(0, T ; H^{1}\left(\Omega_{0}^{\varepsilon}\right)\right), \tilde{c}^{\varepsilon} \rightarrow c^{0}$ in $L^{2}\left(\Omega_{0}^{\varepsilon}\right)$ as $t \rightarrow 0$, and $\tilde{\rho}^{\varepsilon}$ is the piecewise linear interpolation of $\rho_{i}^{\varepsilon} \in H^{1}(0, T)$ defined by $(2.2)$.

To derive a priori estimates, we now study the properties of the coefficients appearing in (4.5). Note that thanks to Proposition $4.1 v^{\varepsilon}$ is bounded above and below independently of $t$ and $\varepsilon$. From the uniform boundedness of $\partial_{t} \tilde{\rho}^{\varepsilon}, 0<\partial_{t} \tilde{\rho}^{\varepsilon}<K$, we deduce that $b^{\varepsilon}=\mathcal{J}^{-1} \partial_{2} \varphi\left(\rho^{0}, \tilde{\rho}^{\varepsilon}, x_{3}\right) \partial_{t} \tilde{\rho}^{\varepsilon}$ is bounded above and below independently of $t$ and $\varepsilon$. However, it is not possible to bound $M$ above and below independently of $V\left(\rho^{\varepsilon}\right)$.

Proposition 4.3. The matrix $M^{\varepsilon}$ is symmetric, positive definite and satisfies for all $\xi \in \mathbb{R}^{3}$, almost all $x \in \Omega, t>0$,

$$
\begin{aligned}
& \frac{1}{\mu} \frac{1}{1+V\left(\rho^{\varepsilon}\right)^{2}}|\xi|^{2} \leq\left(M^{\varepsilon} \xi, \xi\right) \leq \mu\left(1+V\left(\rho^{\varepsilon}\right)^{2}\right)|\xi|^{2} \\
& \left|\left(\partial_{t} M^{\varepsilon} \xi, \xi\right)\right| \leq \mu\left(1+V\left(\rho^{\varepsilon}\right)^{2}+V\left(\partial_{t} \rho^{\varepsilon}\right)^{2}\right)|\xi|^{2}
\end{aligned}
$$

for some positive constant $\mu$ independent of $\varepsilon$ and $t$.

Proof. A straightforward calculation shows that one eigenvalue of $\left(\mathcal{J}^{\varepsilon} / D^{\varepsilon}\right) M^{\varepsilon}$ is equal to 1 , a second eigenvalue is bounded above by $1+\left|\partial_{x_{1}} f^{\varepsilon}\right|^{2}+\left|\partial_{x_{2}} f^{\varepsilon}\right|^{2}+\left|\mathcal{J}^{\varepsilon}\right|^{2}$, and below by $\left|\partial_{x_{3}} f^{\varepsilon}\right|^{2}$, whereas the third is bounded above by $\left|\partial_{x_{3}} f^{\varepsilon}\right|^{2}$ and below by $\left|\mathcal{J}^{\varepsilon}\right|^{2} /\left(1+\left|\partial_{x_{1}} f^{\varepsilon}\right|^{2}+\left|\partial_{x_{2}} f^{\varepsilon}\right|^{2}\right)$. From the bound (4.3) on the oscillations of $\tilde{\rho}^{\varepsilon}$ and the smoothness of $\rho^{0}$ we obtain the first bound. The proof the second bound is similar. We have

$$
\begin{aligned}
\left|\left(\partial_{t} M^{\varepsilon} \xi, \xi\right)\right| \leq & \mu\left(\left|\partial_{t} \tilde{\rho}^{\varepsilon}\right|\|\nabla D\|_{C^{0}(\bar{\Omega})}+\left|\partial_{23} \varphi\right|+\left|\partial_{33} \varphi\right|\right)\left(M^{\varepsilon} \xi, \xi\right) \\
& +\mu\left|\partial_{t}\left(\begin{array}{cll}
1 & 0 & \partial_{x^{\prime}} f^{\varepsilon} \\
0 & 1 & \\
\left(\partial_{x^{\prime}} f^{\varepsilon}\right)^{T} & \left|\partial_{x^{\prime}} f^{\varepsilon}\right|^{2}+\left|\mathcal{J}^{\varepsilon}\right|^{2}
\end{array}\right) \xi \cdot \xi\right| \\
\leq & \mu\left(1+V\left(\rho^{\varepsilon}\right)^{2}+V\left(\partial_{t} \rho^{\varepsilon}\right)^{2}\right)|\xi|^{2},
\end{aligned}
$$

where we used in addition the bounds on the oscillations $\partial_{t} \tilde{\rho}^{\varepsilon}$ and the smooth dependence of $D^{\varepsilon}$ on the macroscopic variable. 
Proposition 4.3 indicates that the horizontal rates of variations $V\left(\rho^{\varepsilon}\right)$ and $V\left(\partial_{t} \rho^{\varepsilon}\right)$ affects the ellipticity of the system. The following proposition bounds these variations in terms of $c^{\varepsilon}$.

Proposition 4.4. The following estimates hold

$$
V\left(\rho^{\varepsilon}\right)+V\left(\partial_{t} \rho^{\varepsilon}\right) \leq \mu\left(\left\|\rho^{0}\right\|_{C^{1}\left([0,1]^{2}\right)}+\left\|\tilde{c}^{\varepsilon}\right\|_{L^{\infty}\left((0, T) \times \Omega_{0}^{\varepsilon}\right)}\right), \quad \text { for } t \in(0, T), x \in(0,1)^{2},
$$

where $\mu$ is a universal constant.

Proof. Note that system (2.2) can be integrated in an implicit form, namely

$$
\rho_{i}^{\varepsilon}(t)=K-\left(K-\rho^{0}(i \varepsilon)\right) \exp \left(-\int_{0}^{t} \frac{c s(s, i, \varepsilon)}{\sqrt{1+c s^{2}(s, i, \varepsilon)}} \mathrm{d} s\right) .
$$

This expression is implicit as $c s(s, i, \varepsilon)$ depends on $\rho_{i}^{\varepsilon}$. We thus obtain that

$$
\begin{aligned}
\left|\rho_{i}^{\varepsilon}(t)-\rho_{j}^{\varepsilon}(t)\right| \leq & \left|\rho^{0}(i \varepsilon)-\rho^{0}(j \varepsilon)\right|+K\left|\int_{0}^{t} \frac{c s(s, i, \varepsilon)}{\sqrt{1+c s^{2}(s, i, \varepsilon)}}-\frac{c s(s, j, \varepsilon)}{\sqrt{1+c s^{2}(s, j, \varepsilon)}} \mathrm{d} s\right| \\
\leq & \left\|\rho^{0}\right\|_{C^{1}\left([0,1]^{2}\right)}|i-j| \varepsilon+K \int_{0}^{t}|c s(s, i, \varepsilon)-c s(s, j, \varepsilon)| \mathrm{d} s \\
\leq & \left\|\rho^{0}\right\|_{C^{1}\left([0,1]^{2}\right)}|i-j| \varepsilon \\
& +K t\left\|\tilde{c}^{\varepsilon}\right\|_{L^{\infty}\left((0, T) \times \Omega_{0}^{\varepsilon}\right)}\left(\left|B\left(i \varepsilon, r_{0}\right) \backslash B\left(j \varepsilon, r_{0}\right)\right|+\left|B\left(j \varepsilon, r_{0}\right) \backslash B\left(i \varepsilon, r_{0}\right)\right|\right) .
\end{aligned}
$$

If $|i-j| \leq 2,\left|B\left(i \varepsilon, r_{0}\right) \backslash B\left(j \varepsilon, r_{0}\right)\right| \leq 4 \varepsilon$, therefore

$$
V\left(\rho^{\varepsilon}\right) \leq \mu\left(\left\|\rho^{0}\right\|_{C^{1}\left([0,1]^{2}\right)}+\left\|\tilde{c}^{\varepsilon}\right\|_{L^{\infty}\left((0, T) \times \Omega_{0}^{\varepsilon}\right)}\right) .
$$

The proof is similar for $V\left(\partial_{t} \rho^{\varepsilon}\right)$, using (2.2),

$$
\begin{aligned}
\left|\frac{\mathrm{d}}{\mathrm{d} t} \rho_{i}^{\varepsilon}(t)-\frac{\mathrm{d}}{\mathrm{d} t} \rho_{j}^{\varepsilon}(t)\right| & \leq\left|\rho_{i}^{\varepsilon}(t)-\rho_{j}^{\varepsilon}(t)\right|+K\left|\frac{c s(t, i, \varepsilon)}{\sqrt{1+c s^{2}(t, i, \varepsilon)}}-\frac{c s(t, j, \varepsilon)}{\sqrt{1+c s^{2}(t, j, \varepsilon)}}\right| \\
& \leq\left\|\rho^{0}\right\|_{C^{1}\left([0,1]^{2}\right)}|i-j| \varepsilon+8 \varepsilon K(t+1)\left\|\tilde{c}^{\varepsilon}\right\|_{L^{\infty}\left((0, T) \times \Omega_{0}^{\varepsilon}\right)} .
\end{aligned}
$$

Next, we show that all weak solutions are bounded in appropriate norms by the initial nutrient concentration, and that a non-negative initial condition guarantees that $c^{\varepsilon}$ stays positive for all time, and therefore can be interpreted as a nutrient concentration.

Lemma 4.5. For any weak solution of the problem (4.4), the maximum principle holds, that is,

$$
\left\|\tilde{c}^{\varepsilon}\right\|_{L^{\infty}\left((0, T) \times \Omega_{0}^{\varepsilon}\right)} \leq \max _{\bar{\Omega}}\left(c^{0}\right), \text { and } \tilde{c}^{\varepsilon} \geq 0 .
$$

Furthermore, the following estimates hold

$$
\left\|\tilde{c}^{\varepsilon}\right\|_{L^{\infty}\left((0, T) \times \Omega_{0}^{\varepsilon}\right)}+\left\|\partial_{t} \tilde{c}^{\varepsilon}\right\|_{L^{2}\left(0, T ; L^{2}\left(\Omega_{0}^{\varepsilon}\right)\right)}+\left\|\nabla \tilde{c}^{\varepsilon}\right\|_{L^{\infty}\left(0, T ; L^{2}\left(\Omega_{0}^{\varepsilon}\right)\right)}+\varepsilon^{1 / 2}\left\|\tilde{c}^{\varepsilon}\right\|_{L^{\infty}\left(0, T ; L^{2}\left(\Gamma_{0}^{\varepsilon}\right)\right)} \leq \mu,
$$

where $\mu$ depends on $\left\|c^{0}\right\|_{H^{1}\left(\Omega_{0}^{\varepsilon}\right)},\left\|c^{0}\right\|_{L^{\infty}\left(\Omega_{0}^{\varepsilon}\right)},\left\|\rho^{0}\right\|_{C^{1}\left([0,1]^{2}\right)}$ and $K$ only. 
Proof. Choosing $\tilde{c}^{\varepsilon}$ as a test function in (4.5) we obtain

$$
\begin{aligned}
& \frac{1}{2} \int_{0}^{\tau} \frac{\mathrm{d}}{\mathrm{d} t} \int_{\Omega_{0}^{\varepsilon}}\left|\tilde{c}^{\varepsilon}\right|^{2} v^{\varepsilon} \mathrm{d} x^{\prime} \mathrm{d} w \mathrm{~d} t+\int_{0}^{\tau} \int_{\Omega_{0}^{\varepsilon}}\left(\frac{1}{2}\left|\tilde{c}^{\varepsilon}\right|^{2}\left(-\partial_{t} v^{\varepsilon}\right)+\partial_{w} \tilde{c}^{\varepsilon} b^{\varepsilon} \tilde{c}^{\varepsilon}\right) \mathrm{d} x^{\prime} \mathrm{d} w \mathrm{~d} t \\
& +\int_{0}^{\tau} \int_{\Omega_{0}^{\varepsilon}} M^{\varepsilon} \nabla \tilde{c}^{\varepsilon} \cdot \nabla \tilde{c}^{\varepsilon} \mathrm{d} x^{\prime} \mathrm{d} w \mathrm{~d} t=-\varepsilon \lambda \int_{0}^{\tau} \int_{\Gamma_{0}^{\varepsilon}} v^{\varepsilon}\left|\tilde{c}^{\varepsilon}\right|^{2} \mathrm{~d} \gamma^{\varepsilon} \mathrm{d} t .
\end{aligned}
$$

Note that $\left|\partial_{t} v^{\varepsilon}\right|=\left.\left|\partial_{t} \tilde{\rho}^{\varepsilon} \partial_{23}^{2} \varphi\left(\rho^{0}, \tilde{\rho}^{\varepsilon}, x_{3}\right)\right| \mathcal{J}^{\varepsilon}\right|^{-2} \mid$ is bounded independently of $t$ and $\varepsilon$.

The third term can be estimated by comparing it with the quadratic gradient term, namely

$$
\left|\int_{0}^{\tau} \int_{\Omega_{0}^{\varepsilon}} \partial_{w} \tilde{c}^{\varepsilon} b^{\varepsilon} \tilde{c}^{\varepsilon} \mathrm{d} x^{\prime} \mathrm{d} w \mathrm{~d} t\right| \geq \frac{1}{2} \int_{0}^{\tau} \int_{\Omega_{0}^{\varepsilon}} D^{\varepsilon} \mathcal{J}^{\varepsilon}\left|\partial_{w} \tilde{c}^{\varepsilon}\right|^{2} \mathrm{~d} x^{\prime} \mathrm{d} w \mathrm{~d} t \frac{1}{2} \int_{0}^{\tau} \int_{\Omega_{0}^{\varepsilon}} \frac{1}{D^{\varepsilon} \mathcal{J}^{\varepsilon}}\left|b^{\varepsilon}\right|^{2}\left|\tilde{c}^{\varepsilon}\right|^{2} \mathrm{~d} x^{\prime} \mathrm{d} w \mathrm{~d} t .
$$

To proceed, note that the lower bound on $M^{\varepsilon}$ shown in Proposition 4.3 still holds (with a different $\mu$ ) if $\frac{1}{2} D^{\varepsilon} \mathcal{J}^{\varepsilon}$ is subtracted from $M_{33}^{\epsilon}$. Thus,

$$
-\frac{1}{2} \int_{0}^{\tau} \int_{\Omega_{0}^{\varepsilon}} D^{\varepsilon} \mathcal{J}^{\varepsilon}\left|\partial_{w} \tilde{c}^{\varepsilon}\right|^{2} \mathrm{~d} x^{\prime} \mathrm{d} w \mathrm{~d} t+\int_{0}^{\tau} \int_{\Omega_{0}^{\varepsilon}} M^{\varepsilon} \nabla \tilde{c}^{\varepsilon} \cdot \nabla \tilde{c}^{\varepsilon} \mathrm{d} x^{\prime} \mathrm{d} w \mathrm{~d} t \geq \int_{0}^{\tau} \int_{\Omega_{0}^{\varepsilon}} \frac{1}{\mu\left(1+V\left(\rho^{\varepsilon}\right)^{2}\right)}\left|\nabla \tilde{c}^{\varepsilon}\right|^{2} \mathrm{~d} x^{\prime} \mathrm{d} w \mathrm{~d} t,
$$

and we have obtained

$$
\begin{array}{r}
\int_{\Omega_{0}^{\varepsilon}}\left|\tilde{c}^{\varepsilon}(\tau)\right|^{2} \mathrm{~d} x^{\prime} \mathrm{d} w+\int_{0}^{\tau} \int_{\Omega_{0}^{\varepsilon}} \frac{1}{\mu\left(1+V\left(\rho^{\varepsilon}\right)^{2}\right)}\left|\nabla \tilde{c}^{\varepsilon}\right|^{2} \mathrm{~d} x^{\prime} \mathrm{d} w \mathrm{~d} t+\varepsilon \int_{0}^{\tau} \int_{\Gamma_{0}^{\varepsilon}} v^{\varepsilon}\left|\tilde{c}^{\varepsilon}\right|^{2} \mathrm{~d} \gamma \mathrm{d} t \\
\leq \int_{\Omega_{0}^{\varepsilon}}\left|c^{0}\right|^{2} \mathrm{~d} x+\int_{0}^{\tau} \int_{\Omega_{0}^{\varepsilon}}\left(\frac{1}{\mathcal{J}^{\varepsilon}}\left|b^{\varepsilon}\right|^{2}+\left|\partial_{t} v^{\varepsilon}\right|\right)\left|\tilde{c}^{\varepsilon}\right|^{2} \mathrm{~d} x^{\prime} \mathrm{d} w \mathrm{~d} t .
\end{array}
$$

which, thanks to Gronwall's Lemma implies that

$$
\left\|\tilde{c}^{\varepsilon}\right\|_{L^{\infty}\left(0, T ; L^{2}\left(\Omega_{0}^{\varepsilon}\right)\right)} \leq \mu\left\|c^{0}\right\|_{L^{2}\left(\Omega_{0}^{\varepsilon}\right)}
$$

where $\mu$ is independent of $\varepsilon$. Alternatively, choosing as a test function $\min \left(\tilde{c}^{\varepsilon}, 0\right)$, or $\max \left(\tilde{c}^{\varepsilon}-\alpha, 0\right)$, for any constant $\alpha$ yields, following the same steps,

$$
\left\|\min \left(\tilde{c}^{\varepsilon}, 0\right)\right\|_{L^{\infty}\left(0, T ; L^{2}\left(\Omega_{0}^{\varepsilon}\right)\right)} \leq C\left\|\min \left(c^{0}, 0\right)\right\|_{L^{2}\left(\Omega_{0}^{\varepsilon}\right)}^{2},
$$

and

$$
\left\|\max \left(\tilde{c}^{\varepsilon}-\alpha, 0\right)\right\|_{L^{\infty}\left(0, T ; L^{2}\left(\Omega_{0}^{\varepsilon}\right)\right)} \leq C\left\|\max \left(c^{0}-\alpha, 0\right)\right\|_{L^{2}\left(\Omega_{0}^{\varepsilon}\right)}^{2},
$$

which proves (4.6), choosing $\alpha=\max _{\bar{\Omega}}\left(c^{0}\right)$ in the second case. Using now (4.6) in (4.8), together with the bounds on $V\left(\rho^{\varepsilon}\right)$ given by Proposition 4.4, we have

$$
\int_{\Omega_{0}^{\varepsilon}}\left|\tilde{c}^{\varepsilon}(\tau)\right|^{2} \mathrm{~d} x^{\prime} \mathrm{d} w+\int_{0}^{\tau} \int_{\Omega_{0}^{\varepsilon}}\left|\nabla \tilde{c}^{\varepsilon}\right|^{2} \mathrm{~d} x^{\prime} \mathrm{d} w \mathrm{~d} t+\varepsilon \int_{0}^{\tau} \int_{\Gamma_{0}^{\varepsilon}}\left|\tilde{c}^{\varepsilon}\right|^{2} \mathrm{~d} \gamma^{\varepsilon} \mathrm{d} t \leq \mu\left(1+\int_{0}^{\tau} \int_{\Omega_{0}^{\varepsilon}}\left|\tilde{c}^{\varepsilon}\right|^{2} \mathrm{~d} x^{\prime} \mathrm{d} w \mathrm{~d} t\right),
$$


where $\mu$ is independent of $\varepsilon$. Applying Gronwall's Lemma again shows that

$$
\left\|\nabla \tilde{c}^{\varepsilon}\right\|_{L^{2}\left(0, T ; L^{2}\left(\Omega_{0}^{\varepsilon}\right)\right)}+\left\|\tilde{c}^{\varepsilon}\right\|_{L^{2}\left(0, T ; L^{2}\left(\Gamma_{0}^{\varepsilon}\right)\right)} \leq \mu
$$

where $\mu$ is independent of $\varepsilon$. Using $\partial_{t} \tilde{c}^{\varepsilon}$ as a test function in (4.5) we obtain

$$
\begin{aligned}
& \int_{0}^{\tau} \int_{\Omega_{0}^{\varepsilon}}\left|\partial_{t} \tilde{c}^{\varepsilon}\right|^{2} v^{\varepsilon} \mathrm{d} x^{\prime} \mathrm{d} w \mathrm{~d} t+\frac{1}{2} \int_{0}^{\tau} \frac{\mathrm{d}}{\mathrm{d} t} \int_{\Omega_{0}^{\varepsilon}} M^{\varepsilon} \nabla \tilde{c}^{\varepsilon} \nabla \tilde{c}^{\varepsilon} \mathrm{d} x^{\prime} \mathrm{d} w \mathrm{~d} t-\frac{1}{2} \int_{0}^{\tau} \int_{\Omega_{0}^{\varepsilon}} \partial_{t} M^{\varepsilon} \nabla \tilde{c}^{\varepsilon} \nabla \tilde{c}^{\varepsilon} \mathrm{d} x^{\prime} \mathrm{d} w \mathrm{~d} t \\
& +\int_{0}^{\tau} \int_{\Omega_{0}^{\varepsilon}} b^{\varepsilon} \partial_{w} \tilde{c}^{\varepsilon} \partial_{t} \tilde{c}^{\varepsilon} \mathrm{d} x^{\prime} \mathrm{d} w \mathrm{~d} t=-\varepsilon \frac{\lambda}{2} \int_{0}^{\tau} \frac{\mathrm{d}}{\mathrm{d} t} \int_{\Gamma_{0}^{\varepsilon}} v^{\varepsilon}\left|\tilde{c}^{\varepsilon}\right|^{2} \mathrm{~d} \gamma \mathrm{d} t+\varepsilon \frac{\lambda}{2} \int_{0}^{\tau} \int_{\Gamma_{0}^{\varepsilon}}\left|\tilde{c}^{\varepsilon}\right|^{2} \partial_{t} v^{\varepsilon} \mathrm{d} \gamma \mathrm{d} t .
\end{aligned}
$$

Since $b^{\varepsilon}$ is bounded independently of $t$ and $\varepsilon$, we can write

$$
\int_{0}^{\tau} \int_{\Omega_{0}^{\varepsilon}} b^{\varepsilon} \partial_{w} \tilde{c}^{\varepsilon} \partial_{t} \tilde{c}^{\varepsilon} \mathrm{d} x^{\prime} \mathrm{d} w \mathrm{~d} t \leq \delta \int_{0}^{\tau} \int_{\Omega_{0}^{\varepsilon}}\left|\partial_{t} \tilde{c}^{\varepsilon}\right|^{2} \mathrm{~d} x^{\prime} \mathrm{d} w \mathrm{~d} t+C_{\delta} \int_{0}^{\tau} \int_{\Omega_{0}^{\varepsilon}}\left|\partial_{w} \tilde{c}^{\varepsilon}\right|^{2} \mathrm{~d} x^{\prime} \mathrm{d} w \mathrm{~d} t
$$

with $\delta=\min v^{\varepsilon} / 2$ and $C_{\delta}=\|b\|_{L^{\infty}\left((0, T) \times \Omega_{0}^{\varepsilon}\right)}^{2} /(2 \delta)$. From (4.10), we deduce that the last term is bounded independently of $\varepsilon$ and time, thus, we obtain

$$
\begin{aligned}
& \int_{0}^{\tau} \int_{\Omega_{0}^{\varepsilon}}\left|\partial_{t} \tilde{c}^{\varepsilon}\right|^{2} \mathrm{~d} x^{\prime} \mathrm{d} w \mathrm{~d} t+\frac{1}{2} \int_{0}^{\tau} \frac{\mathrm{d}}{\mathrm{d} t} \int_{\Omega_{0}^{\varepsilon}} M^{\varepsilon} \nabla \tilde{c}^{\varepsilon} \nabla \tilde{c}^{\varepsilon} \mathrm{d} x^{\prime} \mathrm{d} w \mathrm{~d} t+\varepsilon \int_{\Gamma_{0}^{\varepsilon}} v^{\varepsilon}\left|\tilde{c}^{\varepsilon}\right|^{2} \mathrm{~d} \gamma \\
& \leq \mu+\varepsilon \int_{\Gamma_{0}^{\varepsilon}}\left|c^{0}\right|^{2} \mathrm{~d} \gamma+\frac{1}{2} \int_{0}^{\tau} \int_{\Omega_{0}^{\varepsilon}} \partial_{t} M^{\varepsilon} \nabla \tilde{c}^{\varepsilon} \nabla \tilde{c}^{\varepsilon} \mathrm{d} x^{\prime} \mathrm{d} w \mathrm{~d} t \\
& \leq \mu,
\end{aligned}
$$

where we used the bounds on $\partial_{t} M$ given by Proposition 4.3, the $L^{2}$ bound (4.10) and the $L^{\infty}$ bound (4.6). Together with (4.6), this last bound implies (4.7).

Corollary 4.6. The interpolated root length $\tilde{\rho}^{\varepsilon}$, and its time derivative $\partial_{t} \tilde{\rho}^{\varepsilon}$ satisfy

$$
\left\|\nabla_{x^{\prime}} \tilde{\rho}^{\varepsilon}\right\|_{L^{\infty}\left((0, T) \times(0,1)^{2}\right)}+\left\|\nabla_{x^{\prime}} \partial_{t} \tilde{\rho}^{\varepsilon}\right\|_{L^{\infty}\left((0, T) \times(0,1)^{2}\right)} \leq \mu\left(\left\|\rho^{0}\right\|_{C^{1}\left([0,1]^{2}\right)}+\max _{\bar{\Omega}} c^{0}\right),
$$

where $\mu$ is an universal constant.

Proof. Thanks to Proposition 4.4 and the $L^{\infty}$ bound on $\tilde{c}^{\varepsilon}$ given by Lemma 4.5 , we have

$$
V\left(\rho^{\varepsilon}\right)+V\left(\partial_{t} \rho^{\varepsilon}\right) \leq \mu\left(\left\|\rho^{0}\right\|_{C^{1}\left([0,1]^{2}\right)}+\max _{\bar{\Omega}} c^{0}\right), \quad \text { for } t \in(0, T), x \in(0,1)^{2} .
$$

Since $\tilde{\rho}^{\varepsilon}$ linearly interpolates in $x^{\prime}$ between the values of $\rho^{\varepsilon}$, this implies the result.

\subsection{Existence and uniqueness}

We can now state the main result of this section 
Proposition 4.7. For every $\varepsilon>0$, there exists a unique weak solution of (4.4). That solution satisfies the following estimate

$$
\left\|\tilde{c}^{\varepsilon}\right\|_{L^{\infty}\left(0, T ; L^{\infty}\left(\Omega_{0}^{\varepsilon}\right)\right.}+\left\|\partial_{t} \tilde{c}^{\varepsilon}\right\|_{L^{2}\left(0, T ; L^{2}\left(\Omega_{0}^{\varepsilon}\right)\right)}+\left\|\nabla \tilde{c}^{\varepsilon}\right\|_{L^{\infty}\left(0, T ; L^{2}\left(\Omega_{0}^{\varepsilon}\right)\right)}+\varepsilon^{1 / 2}\left\|\tilde{c}^{\varepsilon}\right\|_{L^{\infty}\left((0, T) ; L^{2}\left(\Gamma_{0}^{\varepsilon}\right)\right)} \leq \mu,
$$

where $\mu$ depends on $\left\|c^{0}\right\|_{H^{1}\left(\Omega_{0}^{\varepsilon}\right)},\left\|c^{0}\right\|_{L^{\infty}\left(\Omega_{0}^{\varepsilon}\right)},\left\|\rho^{0}\right\|_{C^{1}\left((0,1)^{2}\right)}$ and $K$ only.

Furthermore, $0 \leq \tilde{c}^{\varepsilon} \leq \max _{\bar{\Omega}}\left(c^{0}\right)$ for all times $t$ and almost all $x \in \Omega_{0}^{\varepsilon}$.

Proof of Proposition 3.1. By performing the inverse change of variable, Proposition 4.7 shows that problem (2.1) is well-posed.

The rest of the section is devoted to the proof of this result. Because the problem is non-linear, we shall first prove existence by a fixed point argument.

Setting $c_{0}^{\varepsilon}(t, x)=c^{0}(x)$, we define for all $n \geq 0$ the interpolated length the root lengths $\rho_{i, n}^{\varepsilon}$, by the following system of differential equations

$$
\begin{gathered}
\frac{\mathrm{d}}{\mathrm{d} t} \rho_{i, n}^{\varepsilon}=\frac{c s(t, i, n, \varepsilon)}{\sqrt{1+c s^{2}(t, i, n, \varepsilon)}}\left(K-\rho_{i, n}^{\varepsilon}\right) \quad \text { for } 1 \leq i \leq N_{\varepsilon}, \\
\rho_{i . n}^{\varepsilon}(0)=\rho^{0}\left(\varepsilon x_{i}^{\prime}\right)
\end{gathered}
$$

where

the integral being calculated on

$$
c s(t, i, n, \varepsilon)=\int_{\Omega_{n, t}^{\varepsilon} \cap B\left(i \varepsilon, r_{0}\right)} c_{n}^{\varepsilon}(t, x) \mathrm{d} x
$$

$$
\Omega_{n, t}^{\varepsilon}=\Omega \backslash\left(\bigcup_{i=1}^{N_{\varepsilon}} S_{i} \times\left(0, \rho_{i, n}^{\varepsilon}(t)\right)\right)
$$

We then define $\tilde{c}_{n+1}^{\varepsilon}$ - thus by a change of variable, $c_{n}^{\varepsilon}$ - as the solution of the linear evolution problem

$$
\begin{array}{ll}
v\left(\tilde{\rho}_{n}^{\varepsilon}\right) \partial_{t} \tilde{c}_{n+1}^{\varepsilon}+b\left(\tilde{\rho}_{n}^{\varepsilon}\right) \partial_{w} \tilde{c}_{n+1}^{\varepsilon}-\nabla \cdot\left(M\left(\tilde{\rho}_{n}^{\varepsilon}\right) \nabla \tilde{c}_{n+1}^{\varepsilon}\right)=0 & \text { in }(0, T) \times \Omega_{0}^{\varepsilon}, \\
M\left(\tilde{\rho}_{n}^{\varepsilon}\right) \nabla \tilde{c}_{n+1}^{\varepsilon} \cdot \nu=-\varepsilon v\left(\tilde{\rho}_{n}^{\varepsilon}\right) \lambda \tilde{c}_{n+1}^{\varepsilon} & \text { on }(0, T) \times \Gamma_{0}^{\varepsilon}, \\
M\left(\tilde{\rho}_{n}^{\varepsilon}\right) \nabla \tilde{c}_{n+1}^{\varepsilon} \cdot \nu=0 & \text { on }(0, T) \times\left(\partial \Omega_{0}^{\varepsilon} \backslash \Gamma_{0}^{\varepsilon}\right), \\
\tilde{c}_{n+1}^{\varepsilon}=c^{0}\left(x^{\prime}, w\right) & \text { in } \Omega_{0}^{\varepsilon}, \text { at } t=0 .
\end{array}
$$

The $\sim$ representing, as before, interpolated quantities. Naturally, if $\left(c_{n}, \rho_{n}\right)=\left(c_{n+1}, \rho_{n+1}\right)$, then $\left(c_{n}, \rho_{n}\right)$ is a solution of (2.1). The existence of weak solutions to (4.4) is given by the following proposition

Proposition 4.8. Given $\varepsilon>0$, up to the possible extraction of a sub-sequence, the sequence $c_{n}^{\varepsilon}$ converges weakly in $H^{1}\left(0, T ; H^{1}\left(\Omega_{0}^{\varepsilon}\right)\right)$ to a weak solution $c^{\varepsilon}$ of $(4.4)$.

Proof. We first verify that

$$
\left(t,\left(\rho_{i, n}^{\varepsilon}\right)_{1 \leq i \leq N_{\varepsilon}}\right) \rightarrow\left(\frac{c s(t, i, n, \varepsilon)}{\sqrt{1+c s^{2}(t, i, n, \varepsilon)}}\left(K-\rho_{i, n}^{\varepsilon}\right)\right)_{1 \leq i \leq N_{\varepsilon}}
$$

is Lipschitz-continuous with respect to $\rho_{i, n}^{\varepsilon}$. Given $\left(\rho_{i, n}^{a, \varepsilon}\right)_{1 \leq i \leq N_{\varepsilon}}$ and $\left(\rho_{i, n}^{b, \varepsilon}\right)_{1 \leq i \leq N_{\varepsilon}}$, we have

$$
\left|\left(\Omega \backslash\left(\bigcup_{i=1}^{N_{\varepsilon}} S_{i} \times\left(0, \rho_{i, n}^{a, \varepsilon}(t)\right)\right)\right) \backslash\left(\Omega \backslash\left(\bigcup_{i=1}^{N_{\varepsilon}} S_{i} \times\left(0, \rho_{i, n}^{b, \varepsilon}(t)\right)\right)\right)\right| \leq \mu_{\varepsilon} \max _{i}\left|\rho_{i, n}^{a, \varepsilon}(t)-\rho_{i, n}^{b, \varepsilon}(t)\right|,
$$


where $\mu_{\varepsilon}$ does not depend on $\rho_{i, n}^{a, \varepsilon}(t)$ or $\rho_{i, n}^{b, \varepsilon}(t)$ (and can be replaced by 1 , since $|\Omega|=1$ ), thus $\mid c s_{a}(t, i, n, \varepsilon)-c s_{b}(t$, $i, n, \varepsilon)\left|\leq\left\|\tilde{c}_{n}^{\varepsilon}\right\|_{L^{\infty}\left((0, T) \times \Omega_{0}^{\varepsilon}\right)} \max _{i}\right| \rho_{i, n}^{a, \varepsilon}(t)-\rho_{i, n}^{b, \varepsilon}(t) \mid$ which is a Lipschitz bound provided $\left\|\tilde{c}_{n}^{\varepsilon}\right\|_{L^{\infty}\left((0, T) \times \Omega_{0}^{\varepsilon}\right)}$ is bounded. Thanks to Lemma 4.5 , we know that for all $n \geq 1$,

$$
\left\|\tilde{c}_{n}^{\varepsilon}\right\|_{L^{\infty}\left((0, T) \times \Omega_{0}^{\varepsilon}\right)} \leq \max _{\bar{\Omega}}\left(c^{0}\right),
$$

and

$$
\left\|\tilde{c}_{n}^{\varepsilon}\right\|_{L^{\infty}\left((0, T) \times \Omega_{0}^{\varepsilon}\right)}+\left\|\partial_{t} \tilde{c}_{n}^{\varepsilon}\right\|_{L^{2}\left(0, T ; L^{2}\left(\Omega_{0}^{\varepsilon}\right)\right)}+\left\|\nabla \tilde{c}_{n}^{\varepsilon}\right\|_{L^{\infty}\left(0, T ; L^{2}\left(\Omega_{0}^{\varepsilon}\right)\right)}+\left\|\tilde{c}_{n}^{\varepsilon}\right\|_{L^{\infty}\left(0, T ; L^{2}\left(\Gamma_{0}^{\varepsilon}\right)\right)} \leq \mu,
$$

where $\mu$ is independent of $n$, thus the Lipschitz bound is valid for all $n$. Additionally there exists a sub-sequence, still indexed by $n$, converging weakly to a limit $\tilde{c}^{\varepsilon}$ in $L^{\infty}\left(0, T ; H^{1}\left(\Omega_{0}^{\varepsilon}\right)\right) \cap H^{1}\left(0, T ; L^{2}\left(\Omega_{0}^{\varepsilon}\right)\right)$. In particular this convergence is strong in $L^{2}\left((0, T) \times \Omega_{0}^{\varepsilon}\right)$. From the implicit formula

$$
\rho_{i, n}^{\varepsilon}(t)=K-\left(K-\rho^{0}(i \varepsilon)\right) \exp \left(-\int_{0}^{t} \frac{c s(s, i, \varepsilon, n)}{\sqrt{1+c s^{2}(s, i, \varepsilon, n)}} \mathrm{d} s\right)
$$

we deduce that any given $n, m \geq 0$ we have

$$
\begin{aligned}
\left|\rho_{i, n}^{\varepsilon}(t)-\rho_{i, m}^{\varepsilon}(t)\right| & \leq K\left|\exp \left(-\int_{0}^{t} \frac{c s(s, i, \varepsilon, n)}{\sqrt{1+c s^{2}(s, i, \varepsilon, n)}} \mathrm{d} s\right)-\exp \left(-\int_{0}^{t} \frac{c s(s, i, \varepsilon, m)}{\sqrt{1+c s^{2}(s, i, \varepsilon, m)}} \mathrm{d} s\right)\right| \\
& \leq K\left|\int_{0}^{t} \frac{c s(s, i, \varepsilon, n)}{\sqrt{1+c s^{2}(s, i, \varepsilon, n)}}-\frac{c s(s, i, \varepsilon, m)}{1+c s^{2}(s, i, \varepsilon, m)} \mathrm{d} s\right| \\
& \leq K\left|\int_{0}^{t}\right| c s(s, i, \varepsilon, n)-c s(s, i, \varepsilon, m, s)|\mathrm{d} s| \\
& \leq \frac{K}{1-K} \int_{0}^{t} \int_{\Omega_{0}^{\varepsilon}}\left|\tilde{c}_{n}^{\varepsilon}-\tilde{c}_{m}^{\varepsilon}\right| \mathrm{d} x \mathrm{~d} s+K \max _{\bar{\Omega}}\left(c^{0}\right) \max _{i} \int_{0}^{t}\left|\rho_{i, n}^{\varepsilon}(t)-\rho_{i, m}^{\varepsilon}(t)\right| \mathrm{d} t
\end{aligned}
$$

where for the last inequality we used Proposition 4.1 and the Lipschitz bound derived above. Therefore, thanks to Gronwall's Lemma, for all $t \in[0, T]$,

$$
\max _{i}\left|\rho_{i, n}^{\varepsilon}(t)-\rho_{i, m}^{\varepsilon}(t)\right| \leq \mu\left\|\tilde{c}_{n}^{\varepsilon}-\tilde{c}_{m}^{\varepsilon}\right\|_{L^{1}\left((0, T) \times \Omega_{0}^{\varepsilon}\right)},
$$

where $\mu$ is a constant independent of $n, m$ and $t$. This means that

$$
\lim _{n \rightarrow \infty}\left\|\tilde{\rho}_{n}^{\varepsilon}-\tilde{\rho}^{\varepsilon}\right\|_{L^{\infty}\left((0, T) \times[0,1]^{2}\right)}=0,
$$

and in turn that, due to continuity of $v, b, f, \mathcal{J}$ and $M$,

$$
\left(v\left(\tilde{\rho}_{n}^{\varepsilon}\right), b\left(\tilde{\rho}_{n}^{\varepsilon}\right), f\left(\tilde{\rho}_{n}^{\varepsilon}, \cdot\right), \mathcal{J}\left(\tilde{\rho}_{n}^{\varepsilon}\right), M\left(\tilde{\rho}_{n}^{\varepsilon}\right)\right) \rightarrow\left(v\left(\tilde{\rho}^{\varepsilon}\right), b\left(\tilde{\rho}^{\varepsilon}\right), f\left(\tilde{\rho}^{\varepsilon}, \cdot\right), \mathcal{J}\left(\tilde{\rho}^{\varepsilon}\right), M\left(\tilde{\rho}^{\varepsilon}\right)\right)
$$

the convergence being strong in $L^{\infty}\left((0, T) \times \Omega_{0}^{\varepsilon}\right)$. We can therefore safely pass to the limit as $n$ tends to infinity in the weak formulation of problem (4.12), and obtain that the limit $c^{\varepsilon}$ satisfies (4.4).

The proof of Proposition 4.7 will be complete once we show that the solution is unique, which is the subject of the next proposition. 
Proposition 4.9. Given $\varepsilon>0$, the weak solution $\tilde{c}^{\varepsilon} \in L^{2}\left(0, T ; H^{1}\left(\Omega_{0}^{\varepsilon}\right)\right)$, $\partial_{t} \tilde{c}^{\varepsilon} \in L^{2}\left(0, T ; L^{2}\left(\Omega_{0}^{\varepsilon}\right)\right)$ to Problem (4.4) is unique.

Proof. Suppose there are two solution, the first one indexed by a and the second by b, e.g. $c_{a}^{\varepsilon}$ and $c_{b}^{\varepsilon}$. From the implicit equation

$$
\rho_{i}^{\varepsilon}(t)=K-\left(K-\rho^{0}(i \varepsilon)\right) \exp \left(-\int_{0}^{t} \frac{c s(i, \varepsilon, s)}{\sqrt{1+c s^{2}(i, \varepsilon, s)}} \mathrm{d} s\right), \quad t \in(0, T),
$$

following the same steps as in the derivation of the bound (4.13) we obtain

$$
\left\|\tilde{\rho}_{a}^{\varepsilon}-\tilde{\rho}_{b}^{\varepsilon}\right\|_{L^{\infty}\left((0, t) \times(0,1)^{2}\right)} \leq \mu\left\|\tilde{c}_{a}^{\varepsilon}-\tilde{c}_{b}^{\varepsilon}\right\|_{L^{1}\left((0, t) \times \Omega_{0}^{\varepsilon}\right)}
$$

for a constant $\mu$ independent of $\tilde{c}_{a}^{\varepsilon}, \tilde{c}_{b}^{\varepsilon}, t$ and $\varepsilon$, and this in turn implies

$$
\left|v\left(\tilde{\rho}_{a}^{\varepsilon}\right)-v\left(\tilde{\rho}_{b}^{\varepsilon}\right)\right|+\left|b\left(\tilde{\rho}_{a}^{\varepsilon}\right)-b\left(\tilde{\rho}_{b}^{\varepsilon}\right)\right|+\left|M\left(\tilde{\rho}_{a}^{\varepsilon}\right)-M\left(\tilde{\rho}_{b}^{\varepsilon}\right)\right| \leq \mu^{\varepsilon}\left\|\tilde{c}_{a}^{\varepsilon}-\tilde{c}_{b}^{\varepsilon}\right\|_{L^{1}\left((0, t) \times \Omega_{0}^{\varepsilon}\right)},
$$

where $\mu^{\varepsilon}$ depends on $\varepsilon$, but is independent of $\tilde{c}_{a}^{\varepsilon}, \tilde{c}_{b}^{\varepsilon}$ and $t$. Comparing the weak variational formulations (4.5) defining $\tilde{c}_{a}^{\varepsilon}$ and $\tilde{c}_{b}^{\varepsilon}$, we have

$$
\begin{aligned}
& \int_{0} \int_{\Omega_{0}^{\varepsilon}}\left(\left(v\left(\tilde{\rho}_{a}^{\varepsilon}\right)+v\left(\tilde{\rho}_{b}^{\varepsilon}\right)\right) \partial_{t}\left(\tilde{c}_{a}^{\varepsilon}-\tilde{c}_{b}^{\varepsilon}\right) \phi+\left(b\left(\tilde{\rho}_{a}^{\varepsilon}\right)+b\left(\tilde{\rho}_{b}^{\varepsilon}\right)\right) \partial_{w}\left(\tilde{c}_{a}^{\varepsilon}-\tilde{c}_{b}^{\varepsilon}\right) \phi\right) \mathrm{d} x^{\prime} \mathrm{d} w \mathrm{~d} t \\
& +\int_{0}^{\tau} \int_{\Omega_{0}^{\varepsilon}}\left(\left(M\left(\tilde{\rho}_{a}^{\varepsilon}\right)+M\left(\tilde{\rho}_{b}^{\varepsilon}\right)\right) \nabla\left(\tilde{c}_{a}^{\varepsilon}-\tilde{c}_{b}^{\varepsilon}\right) \nabla \phi\right) \mathrm{d} x^{\prime} \mathrm{d} w \mathrm{~d} t \\
& +\varepsilon \int_{0}^{\tau} \int_{\Gamma_{0}^{\varepsilon}} \lambda\left(v\left(\tilde{\rho}_{a}^{\varepsilon}\right)+v\left(\tilde{\rho}_{b}^{\varepsilon}\right)\right)\left(\tilde{c}_{a}^{\varepsilon}-\tilde{c}_{b}^{\varepsilon}\right) \phi \mathrm{d} \gamma^{\varepsilon} \mathrm{d} t \\
& =-\int_{0}^{\tau} \int_{\Omega_{0}^{\varepsilon}}\left(\left(v\left(\tilde{\rho}_{a}^{\varepsilon}\right)-v\left(\tilde{\rho}_{b}^{\varepsilon}\right)\right) \partial_{t}\left(\tilde{c}_{a}^{\varepsilon}+\tilde{c}_{b}^{\varepsilon}\right) \phi+\left(b\left(\tilde{\rho}_{a}^{\varepsilon}\right)-b\left(\tilde{\rho}_{b}^{\varepsilon}\right)\right) \partial_{w}\left(\tilde{c}_{a}^{\varepsilon}+\tilde{c}_{b}^{\varepsilon}\right) \phi\right) \mathrm{d} x^{\prime} \mathrm{d} w \mathrm{~d} t \\
& -\int_{0}^{\tau} \int_{\Omega_{0}^{\varepsilon}}\left(\left(M\left(\tilde{\rho}_{a}^{\varepsilon}\right)-M\left(\tilde{\rho}_{b}^{\varepsilon}\right)\right) \nabla\left(\tilde{c}_{a}^{\varepsilon}+\tilde{c}_{b}^{\varepsilon}\right) \nabla \phi\right) \mathrm{d} x^{\prime} \mathrm{d} w \mathrm{~d} t \\
& -\varepsilon \int_{0}^{\tau} \int_{\Gamma_{0}^{\varepsilon}} \lambda\left(v\left(\tilde{\rho}_{a}^{\varepsilon}\right)-v\left(\tilde{\rho}_{b}^{\varepsilon}\right)\right)\left(\tilde{c}_{a}^{\varepsilon}+\tilde{c}_{b}^{\varepsilon}\right) \phi \mathrm{d} \gamma^{\varepsilon} \mathrm{d} t .
\end{aligned}
$$

Combining the a priori bounds (4.11) satisfied by both $c_{a}^{\varepsilon}$ and $c_{\varepsilon}^{b}$ and (4.15), we see that the right hand side of the above expression is bounded from above by

$$
\mu^{\varepsilon}\left\|c_{a}^{\varepsilon}-c_{b}^{\varepsilon}\right\|_{L^{1}\left((0, \tau) \times \Omega_{0}^{\varepsilon}\right)}\left(\|\nabla \phi\|_{L^{2}\left((0, \tau) \times \Omega_{0}^{\varepsilon}\right)}+\|\phi\|_{L^{2}\left((0, \tau) \times \Omega_{0}^{\varepsilon}\right)}+\sqrt{\varepsilon}\|\phi\|_{L^{2}\left((0, \tau) \times \Gamma_{0}^{\varepsilon}\right)}\right)
$$


where $\mu^{\varepsilon}$ is independent of $c_{a}^{\varepsilon}, c_{b}^{\varepsilon}$ and $\tau$. On the other hand, taking $\phi=c_{a}^{\varepsilon}-c_{b}^{\varepsilon}$ and arguing as in Lemma 4.5, the left hand side is bounded from below by

$$
\begin{aligned}
& \mu\left(\int_{\Omega_{0}^{\varepsilon}}\left|\tilde{c}_{a}^{\varepsilon}(\tau)-\tilde{c}_{b}^{\varepsilon}(\tau)\right|^{2} \mathrm{~d} x^{\prime} \mathrm{d} w+\int_{0}^{\tau} \int_{\Omega_{0}^{\varepsilon}}\left|\nabla\left(\tilde{c}_{a}^{\varepsilon}-\tilde{c}_{b}^{\varepsilon}\right)\right|^{2} \mathrm{~d} x^{\prime} \mathrm{d} w \mathrm{~d} t+\varepsilon \int_{0}^{\tau} \int_{\Gamma_{0}^{\varepsilon}}\left|\tilde{c}_{a}^{\varepsilon}-\tilde{c}_{b}^{\varepsilon}\right|^{2} \mathrm{~d} \gamma \mathrm{d} t\right) \\
& -\frac{1}{\mu} \int_{0}^{\tau} \int_{\Omega_{0}^{\varepsilon}}\left|\tilde{c}_{a}^{\varepsilon}-\tilde{c}_{b}^{\varepsilon}\right|^{2} \mathrm{~d} x^{\prime} \mathrm{d} w \mathrm{~d} t
\end{aligned}
$$

where $\mu$ is independent of $c_{a}^{\varepsilon}, c_{b}^{\varepsilon}$ and $\tau$. Thus, altogether,

$$
\begin{aligned}
& \frac{\mu}{2}\left(\int_{\Omega_{0}^{\varepsilon}}\left|\tilde{c}_{a}^{\varepsilon}(\tau)-\tilde{c}_{b}^{\varepsilon}(\tau)\right|^{2} \mathrm{~d} x^{\prime} \mathrm{d} w+\int_{0}^{\tau} \int_{\Omega_{0}^{\varepsilon}}\left|\nabla\left(\tilde{c}_{a}^{\varepsilon}-\tilde{c}_{b}^{\varepsilon}\right)\right|^{2} \mathrm{~d} x^{\prime} \mathrm{d} w \mathrm{~d} t+\varepsilon \int_{0}^{\tau} \int_{\Gamma_{0}^{\varepsilon}}\left|\tilde{c}_{a}^{\varepsilon}-\tilde{c}_{b}^{\varepsilon}\right|^{2} \mathrm{~d} \gamma \mathrm{d} t\right) \\
& \leq \frac{1}{\mu} \int_{0}^{\tau} \int_{\Omega_{0}^{\varepsilon}}\left|\tilde{c}_{a}^{\varepsilon}-\tilde{c}_{b}^{\varepsilon}\right|^{2} \mathrm{~d} x^{\prime} \mathrm{d} w \mathrm{~d} t+\frac{\left(\mu^{\varepsilon}\right)^{2}}{2 \mu}\left\|c_{a}^{\varepsilon}-c_{b}^{\varepsilon}\right\|_{L^{1}\left((0, \tau) \times \Omega_{0}^{\varepsilon}\right)}^{2} .
\end{aligned}
$$

So in particular

$$
\int_{\Omega_{0}^{\varepsilon}}\left|\tilde{c}_{a}^{\varepsilon}(\tau)-\tilde{c}_{b}^{\varepsilon}(\tau)\right|^{2} \mathrm{~d} x^{\prime} \mathrm{d} w \leq \mu^{\varepsilon} \int_{0}^{\tau} \int_{\Omega_{0}^{\varepsilon}}\left|\tilde{c}_{a}^{\varepsilon}-\tilde{c}_{b}^{\varepsilon}\right|^{2} \mathrm{~d} x^{\prime} \mathrm{d} w \mathrm{~d} t
$$

where $\mu^{\varepsilon}$ again represent a constant independent of $c_{a}^{\varepsilon}, c_{b}^{\varepsilon}$ and $\tau$. Thanks to Gronwall's Lemma, this implies that $\tilde{c}_{a}^{\varepsilon}=\tilde{c}_{b}^{\varepsilon}$.

\section{Convergence to a limit problem by homogenization}

In this section, we show that the weak solution $\left(c^{\varepsilon}, \rho_{i}^{\varepsilon}\right)$ of (2.1)-(2.2) converges to a solution of a limit problem independent of $\varepsilon$. As we will see, the limit problem has a unique solution. It is therefore sufficient to prove the result for a sub-sequence.

\subsection{Existence of weak and two-scale limits}

To derive a limit model, we first extend the domain of definition of the nutrient concentration $\tilde{c}^{\varepsilon}$ from $\Omega_{0}^{\varepsilon}$ to $\Omega$, using classical results $[1,8]$.

Lemma 5.1. There exists an extension $\overline{\tilde{c}^{\varepsilon}}$ of $\tilde{c}^{\varepsilon}$ from $H^{1}\left(\Omega_{0}^{\varepsilon}\right)$ into $H^{1}(\Omega)$ such that

$$
\left\|\overline{\tilde{c}^{\varepsilon}}\right\|_{L^{2}(\Omega)} \leq \mu\left\|\tilde{c}^{\varepsilon}\right\|_{L^{2}\left(\Omega_{0}^{\varepsilon}\right)}, \text { and }\left\|\nabla \overline{\tilde{c}}^{\varepsilon}\right\|_{L^{2}(\Omega)} \leq \mu\left\|\nabla \tilde{c}^{\varepsilon}\right\|_{L^{2}\left(\Omega_{0}^{\varepsilon}\right)},
$$

where the constant $\mu$ depends on $Y, Z$ and $\left\|\rho_{0}\right\|_{C\left([0,1]^{2}\right)}$ only.

Remark. Note that the root stems do not intersect the boundary of the periodic cell, and therefore classical extension results $[1,8]$ apply. Note in particular, that near the boundary $\partial \Omega_{0}^{\varepsilon} \cap\left\{x_{3}=0\right\}$ it is suffices to extend $c^{\varepsilon}$ by reflection in the horizontal direction.

For $\tilde{c}^{\varepsilon} \in L^{2}\left(0, T ; H^{1}\left(\Omega_{0}^{\varepsilon}\right)\right) \cap H^{1}\left(0, T ; L^{2}\left(\Omega_{0}^{\varepsilon}\right)\right)$ we define $\hat{c}^{\varepsilon}(\cdot, t):=\overline{\tilde{c}}^{\varepsilon}(\cdot, t)$ almost everywhere in time. Since the extension operator is linear and bounded and $\Omega_{0}^{\varepsilon}$ does not depend on $t$ we obtain that $\hat{c}^{\varepsilon} \in L^{2}\left(0, T ; H^{1}(\Omega)\right) \cap H^{1}\left(0, T ; L^{2}(\Omega)\right)$ and additionally

$$
\left\|\partial_{t} \hat{c}^{\varepsilon}\right\|_{L^{2}((0, T) \times \Omega)} \leq \mu\left\|\partial_{t} \tilde{c}^{\varepsilon}\right\|_{L^{2}\left((0, T) \times \Omega_{0}^{\varepsilon}\right)} .
$$


In the sequel, we identify $\tilde{c}^{\varepsilon}$ with its extension. Since $\tilde{c}^{\varepsilon}$ is now defined on $\Omega$, and with appropriately bounded partial derivatives thanks Lemma 4.5, passing to the limit (up to a possible sub-sequence) is quite standard in homogenization theory, using, for example, the notion of two-scale convergence (see [2,3,14]). We distinguish between the two-scale convergence in a domain and two-scale convergence of a sequence defined on the boundary of the micro-structure. The $\varepsilon$-scaling in the two-scale convergence on the oscillating boundary $\tilde{\Gamma}_{0}^{\varepsilon}=\cup_{i=0}^{N(\varepsilon)}\left(0, \rho^{0}(i \varepsilon)\right) \times \partial S_{i}^{\varepsilon}$ is essential, since the surface area of $\tilde{\Gamma}_{0}^{\varepsilon}$ is of order $\varepsilon^{-1}$.

Lemma 5.2. There exist functions $\tilde{c}, \tilde{c}_{1}$, $\tilde{\rho}$ and a sub-sequences of $\left(\tilde{c}^{\varepsilon}\right)$ and $\left(\rho^{\varepsilon}\right)$ (denoted again by $\left(\tilde{c}^{\varepsilon}\right)$, $\left(\rho^{\varepsilon}\right)$ ) such that

$$
\begin{aligned}
& \tilde{c}^{\varepsilon} \rightarrow \tilde{c} \text { weakly in } L^{2}\left(0, T, H^{1}(\Omega)\right), \quad \partial_{t} \tilde{c}^{\varepsilon} \rightarrow \partial_{t} \tilde{c} \text { weakly in } L^{2}\left(0, T ; L^{2}(\Omega)\right), \\
& \tilde{c}^{\varepsilon} \rightarrow \tilde{c} \text { strongly in } L^{2}\left(0, T ; H^{s}(\Omega)\right), 1 / 2<s<1, \\
& \lim _{\varepsilon \rightarrow 0} \varepsilon\left\|\tilde{c}^{\varepsilon}-c\right\|_{L^{2}\left((0, T) \times \tilde{\Gamma}_{0}^{\varepsilon}\right)}^{2}=0, \\
& \partial_{t} \tilde{c}^{\varepsilon} \rightarrow \partial_{t} \tilde{c} \text { in the sense of two-scale convergence, } \\
& \nabla \tilde{c}^{\varepsilon} \rightarrow \nabla \tilde{c}+\nabla_{y} \tilde{c}_{1} \text { in the sense of two-scale convergence, with } \tilde{c}_{1} \in L^{2}\left((0, T) \times \Omega ; H_{\#}^{1}(Z)\right) \text {, } \\
& \tilde{\rho}^{\varepsilon} \rightarrow \rho \text { strongly in } L^{\infty}\left((0, T) \times(0,1)^{2}\right), \\
& \partial_{t} \tilde{\rho}^{\varepsilon} \rightarrow \partial_{t} \rho \text { strongly in } L^{2}\left(0, T ; L^{\infty}\left((0,1)^{2}\right)\right),
\end{aligned}
$$

where $H_{\#}^{1}(Z)=\left\{f \in H^{1}(Z)\right.$ s.t. $f$ is $Z$-periodic $\}$.

Furthermore, $\tilde{\rho}^{\varepsilon} \rightarrow \rho$ uniformly in $C^{0}\left([0, T] \times[0,1]^{2}\right)$.

Proof. The weak convergence of a sub-sequence follows from a priori estimates in Lemma 4.5. The strong convergence of $\tilde{c}^{\varepsilon}$ in $L^{2}\left(0, T ; H^{s}(\Omega)\right)$ is due to the Aubin-Lions Lemma and the compact embedding of $H^{1}(\Omega)$ in $H^{s}(\Omega)$ for $1 / 2<s<1$. The convergence of $L^{2}\left((0, T) \times \tilde{\Gamma}_{0}^{\varepsilon}\right)$-Norm follows from the strong convergence of $\tilde{c}^{\varepsilon}$ in $L^{2}\left(0, T ; H^{s}(\Omega)\right)$, definition of the $H^{s}$-norm and a standard scaling argument, see [13]. Since $\tilde{c}^{\varepsilon}$ is bounded in $L^{2}\left(0, T ; H^{1}(\Omega)\right)$ and $\partial_{t} \tilde{c}^{\varepsilon}$ is bounded in $L^{2}\left(0, T ; L^{2}(\Omega)\right)$, the compactness theorem for two-scale convergence implies the convergence (a for sub-sequence) of $\tilde{c}^{\varepsilon}, \partial_{t} \tilde{c}^{\varepsilon}$, and $\nabla \tilde{c}^{\varepsilon}$ in the two-scale sense.

To show the strong convergence of $\tilde{\rho}^{\varepsilon}$, we argue the proof of Proposition 4.9 and (4.14) becomes

$$
\left|\rho_{i}^{\varepsilon_{m}}(t)-\rho_{i}^{\varepsilon_{n}}(t)\right| \leq \mu\left\|\tilde{c}^{\varepsilon_{m}}-\tilde{c}^{\varepsilon_{n}}\right\|_{L^{1}\left((0, t) \times \Omega_{0}^{\varepsilon}\right)}
$$

where the constant $\mu$ is independent of $i, \varepsilon_{n}, \varepsilon_{m}$ and $t$. Thus the strong convergence of $\tilde{c}^{\varepsilon}$ in $L^{2}((0, T) \times \Omega)$ implies the strong convergence of $\tilde{\rho}^{\varepsilon}$ to $\rho$ in $L^{\infty}((0, T) \times \Omega)$. The strong convergence of $\partial_{t} \tilde{\rho}^{\varepsilon}$ follows from the ode (2.2) defining $\rho^{\varepsilon}$ and from the strong convergences of $\tilde{\rho}^{\varepsilon}$ and $\tilde{c}^{\varepsilon}$. Thanks to Corollary 4.6 and Arzela-Ascoli Theorem, the convergence of $\tilde{\rho}^{\varepsilon}$ is in fact uniform in the sense of continuous functions on $C^{0}\left([0, T] \times[0,1]^{2}\right)$.

\subsection{Proof of Theorem 3.2.}

In this section, we will show that the solution of the microscopic model (2.1)-(2.3) converges to the unique solution $\rho \in W^{1, \infty}\left(0, T ; L^{\infty}\left((0,1)^{2}\right)\right), c \in L^{2}\left(0, T ; H^{1}(\Omega)\right) \cap H^{1}\left(0, T ; L^{2}(\Omega)\right)$ of the system

$$
\begin{aligned}
& |Y| \partial_{t} c_{1}-\nabla \cdot\left(D(x)(d *+|Y|) \nabla c_{1}\right)+\lambda|\partial S| c_{1} \quad=0 \quad \text { in } \Omega \cap\left\{x: 0<x_{3}<\rho\left(t, x^{\prime}\right)\right\}, \\
& \partial_{t} c_{2}-\nabla \cdot\left(D(x) \nabla c_{2}\right)=0 \quad \text { in } \Omega \cap\left\{x: \rho\left(t, x^{\prime}\right)<x_{3}<1\right\}, \\
& c_{1}-c_{2}=0 \text {, and }(d *+|Y|) \nabla c_{1} \cdot \nu-\nabla c_{2} \cdot \nu=0 \quad \text { on }\left\{x_{3}=\rho\left(t, x^{\prime}\right)\right\} \text {, } \\
& D(x) \nabla c_{1} \cdot \nu=0 \quad \text { on } \partial \Omega \cap\left\{x: 0<x_{3}<\rho\left(t, x_{1}, x_{2}\right)\right\}, \\
& D(x) \nabla c_{2} \cdot \nu=0 \quad \text { on } \partial \Omega \cap\left\{x: \rho\left(t, x_{1}, x_{2}\right)<x_{3}<1\right\} \text {, } \\
& c(t=0, x)=c^{0} \quad \text { in } \Omega_{0} \text {, }
\end{aligned}
$$

where $c=c_{1}$ in $\Omega \cap\left\{x: 0<x_{3}<\rho\left(t, x_{1}, x_{2}\right)\right\}$ and $c=c_{2}$ in $\Omega \cap\left\{x: \rho\left(t, x_{1}, x_{2}\right)<x_{3}<1\right\}$. 


\section{Convergence.}

Due to the strong convergences of $\tilde{\rho}^{\varepsilon}$ and $\tilde{c}^{\varepsilon}$ we obtain that $\rho$ satisfies the equation

$$
\partial_{t} \rho=\frac{\tilde{c s}\left(t, x^{\prime}\right)}{\sqrt{1+\tilde{c s}^{2}\left(t, x^{\prime}\right)}}(K-\rho) \quad \text { in }(0, T) \times(0,1)^{2},
$$

with

$$
\tilde{c s}\left(t, x^{\prime}\right)=\int_{\Omega \cap B\left(\left(x^{\prime}, \rho^{0}\left(x^{\prime}\right)\right), r_{0}\right)} \tilde{c}\left(t, x^{\prime}, w\right) v(\rho) \mathrm{d} x^{\prime} \mathrm{d} w
$$

To derive the macroscopic equation for $c$ we rewrite equation (4.5) in the following weak form

$$
\int_{0}^{T} \int_{\Omega} \chi_{\Omega_{0}^{\varepsilon}}\left(v\left(\tilde{\rho}^{\varepsilon}\right) \partial_{t} \tilde{c}^{\varepsilon} \phi+b\left(\tilde{\rho}^{\varepsilon}\right) \partial_{w} \tilde{c}^{\varepsilon} \phi+M\left(\tilde{\rho}^{\varepsilon}\right) \nabla \tilde{c}^{\varepsilon} \nabla \phi\right) \mathrm{d} x^{\prime} \mathrm{d} w \mathrm{~d} t=-\varepsilon \int_{0}^{T} \int_{\Gamma_{0}^{\varepsilon}} \lambda v\left(\tilde{\rho}^{\varepsilon}\right) \tilde{c}^{\varepsilon} \phi \mathrm{d} \gamma^{\varepsilon} \mathrm{d} t .
$$

To pass to the two-scale limit, we choose a test function $\phi=\psi_{1}(t, x)+\varepsilon \psi_{2}\left(t, x, \frac{x^{\prime}}{\varepsilon}\right)$ and integrate by parts. Using the uniform convergence of $\tilde{\rho}^{\varepsilon}$, the two-scale convergence of $\tilde{c}^{\varepsilon}, \partial_{t} \tilde{c}^{\varepsilon}$ and $\nabla \tilde{c}^{\varepsilon}$, the strong two-scale convergence of $\chi_{\Omega_{0}^{\varepsilon}}$, and the continuity of the coefficients $v, b$ and $M$, we obtain

$$
\begin{aligned}
\int_{0}^{T} \int_{\Omega} \chi_{\Omega_{0}^{\varepsilon}} v\left(\tilde{\rho}^{\varepsilon}\right) \partial_{t} \tilde{c}^{\varepsilon}\left(\psi_{1}(t, x)+\varepsilon \psi_{2}\left(t, x, \frac{x^{\prime}}{\varepsilon}\right)\right) \mathrm{d} x^{\prime} \mathrm{d} w \mathrm{~d} t \rightarrow \int_{0}^{T} \int_{\Omega}\left(|Y| \chi_{\Omega_{0}^{1}}+\chi_{\Omega_{0}^{2}}\right) \partial_{t} \tilde{c} \psi_{1}(t, x) v(\rho) \mathrm{d} x^{\prime} \mathrm{d} w \mathrm{~d} t, \\
\int_{0}^{T} \int_{\Omega} \chi_{\Omega_{0}^{\varepsilon}} b\left(\tilde{\rho}^{\varepsilon}\right) \partial_{w} \tilde{c}^{\varepsilon}\left(\psi_{1}(t, x)+\varepsilon \psi_{2}\left(t, x, \frac{x^{\prime}}{\varepsilon}\right)\right) \mathrm{d} x \mathrm{~d} t \rightarrow \int_{0}^{T} \int_{\Omega}\left(|Y| \chi_{\Omega_{0}^{1}} \partial_{w} \tilde{c}+\chi_{\Omega_{0}^{2}} \partial_{w} \tilde{c}\right) b(\rho) \psi_{1}(t, x) \mathrm{d} x^{\prime} \mathrm{d} w \mathrm{~d} t,
\end{aligned}
$$

and

$$
\begin{aligned}
& \int_{0}^{T} \int_{\Omega} \chi_{\Omega_{0}^{\varepsilon}} M\left(\tilde{\rho}^{\varepsilon}\right) \nabla \tilde{c}^{\varepsilon} \cdot \nabla\left(\psi_{1}+\varepsilon \psi_{2}\right) \mathrm{d} x^{\prime} \mathrm{d} w \mathrm{~d} t \\
& \quad \rightarrow \iint_{0 \Omega \times Y} \chi_{\Omega_{0}^{1}} \tilde{D}(x, w) M(\rho)\left(\nabla \tilde{c}+\nabla_{y} \tilde{c}_{1}\right) \cdot\left(\nabla \psi_{1}+\nabla_{y} \psi_{2}\right) \mathrm{d} y \mathrm{~d} x^{\prime} \mathrm{d} w \mathrm{~d} t \\
& \quad+\int_{0 \Omega \times Z}^{T} \iint_{\Omega_{0}^{2}} \tilde{D}(x, w) M(\rho)\left(\nabla \tilde{c}+\nabla_{y} \tilde{c}_{1}\right) \cdot\left(\nabla \psi_{1}+\nabla_{y} \psi_{2}\right) \mathrm{d} y \mathrm{~d} x^{\prime} \mathrm{d} w \mathrm{~d} t,
\end{aligned}
$$

where $\nabla_{y}=\left(\partial_{y_{1}}, \partial_{y_{2}}, 0\right)^{T}, \Omega_{0}^{1}=\Omega \cap\left\{\left(x^{\prime}, x_{3}\right): 0<x_{3}<\rho^{0}\left(x^{\prime}\right)\right\}$ and $\Omega_{0}^{2}=\Omega \cap\left\{\left(x^{\prime}, x_{3}\right): \rho^{0}\left(x^{\prime}\right)<x_{3}<1\right\}$, and

$$
M(\rho)=\frac{1}{\mathcal{J}(\rho)}\left(\begin{array}{ccl}
1 & 0 & \partial_{x_{1}} f(\rho, \cdot) \\
0 & 1 & \partial_{x_{2}} f(\rho, \cdot) \\
\partial_{x_{1}} f(\rho, \cdot) & \partial_{x_{2}} f(\rho, \cdot) & \left|\partial_{x_{1}} f(\rho, \cdot)\right|^{2}+\left|\partial_{x_{2}} f(\rho, \cdot)\right|^{2}+|\mathcal{J}(\rho)|^{2}
\end{array}\right)
$$


The boundary integral can be rewritten as

$$
\begin{aligned}
& \varepsilon \int_{0}^{T} \int_{\Gamma_{0}^{\varepsilon}} \lambda v\left(\tilde{\rho}^{\varepsilon}\right) \tilde{c}^{\varepsilon}\left(\psi_{1}+\varepsilon \psi_{2}\right) \mathrm{d} \gamma \mathrm{d} t= \\
& \quad \varepsilon \int_{0}^{T} \int_{\tilde{\Gamma}_{0}^{\varepsilon}}\left(\lambda\left(v\left(\tilde{\rho}^{\varepsilon}\right)-v(\rho)\right) \tilde{c}^{\varepsilon}\left(\psi_{1}+\varepsilon \psi_{2}\right)+\lambda v(\rho) \tilde{c}^{\varepsilon}\left(\psi_{1}+\varepsilon \psi_{2}\right)\right) \mathrm{d} \gamma \mathrm{d} t \\
& \quad+\varepsilon \int_{0}^{T} \int_{S_{\rho^{\varepsilon}}^{\varepsilon}} \lambda v\left(\tilde{\rho}^{\varepsilon}\right) \tilde{c}^{\varepsilon}\left(\psi_{1}+\varepsilon \psi_{2}\right) \mathrm{d} \gamma \mathrm{d} t
\end{aligned}
$$

where $\tilde{\Gamma}_{0}^{\varepsilon}=\cup_{i=0}^{N(\varepsilon)}\left(0, \rho^{0}(i \varepsilon)\right) \times \partial S_{i}^{\varepsilon}, S_{\rho^{0}}^{\varepsilon}=\cup_{i=0}^{N(\varepsilon)} S_{i}^{\varepsilon} \times \rho^{0}(i \varepsilon)$ and $\Gamma_{0}^{\varepsilon}=\tilde{\Gamma}_{0}^{\varepsilon} \cup S_{\rho^{0}}^{\varepsilon}$. To show the convergence of the boundary integral over $\tilde{\Gamma}_{0}^{\varepsilon}$ we use the notion of two-scale convergence on the periodic surfaces of the micro-structure, see e.g. $[3,13]$. The surface area of $\tilde{\Gamma}_{0}^{\varepsilon}$ is of order $\varepsilon^{-1}$, thus the $L^{2}$-norm over $\Gamma_{0}^{\varepsilon}$ of the test functions $\psi_{1}$ and $\psi_{2}$ scaled by $\varepsilon^{1 / 2}$ is bounded.

We write

$$
\begin{aligned}
& \varepsilon \int_{0}^{T} \int_{\tilde{\Gamma}_{0}^{\varepsilon}}\left|\lambda\left(v\left(\tilde{\rho}^{\varepsilon}\right)-v(\rho)\right) \tilde{c}^{\varepsilon}\left(\psi_{1}+\varepsilon \psi_{2}\right)\right| \mathrm{d} \gamma^{\varepsilon} \mathrm{d} t \\
& \leq \mu\left\|\tilde{c}^{\varepsilon}\right\|_{L^{\infty}\left((0, T) \times \tilde{\Gamma}_{0}^{\varepsilon}\right)}\left\|\tilde{\rho}^{\varepsilon}-\rho\right\|_{L^{\infty}\left((0, T) \times \partial S^{\varepsilon}\right)}\left(\varepsilon\left\|\psi_{1}\right\|_{L^{2}\left((0, T) \times \tilde{\Gamma}_{0}^{\varepsilon}\right)}^{2}+\varepsilon^{2}\left\|\psi_{2}\right\|_{L^{2}\left((0, T) \times \tilde{\Gamma}_{0}^{\varepsilon}\right)}^{2}\right),
\end{aligned}
$$

and from the uniform boundedness of $\tilde{c}^{\varepsilon}$ and the continuous convergence of $\tilde{\rho}^{\varepsilon}$ to $\rho$ in $\left((0, T) \times \partial S^{\varepsilon}\right)$ we conclude that the right-hand-side tends to zero with $\varepsilon \rightarrow 0$.

For the second integral, we write

$$
\begin{aligned}
& \lambda \varepsilon \int_{0}^{T} \int_{S_{\rho^{0}}^{\varepsilon}}\left|v\left(\tilde{\rho}^{\varepsilon}\right) \tilde{c}^{\varepsilon}\left(\psi_{1}+\varepsilon \psi_{2}\right)\right| \mathrm{d} \gamma \mathrm{d} t \\
& \leq \mu\left\|\tilde{c}^{\varepsilon}\right\|_{L^{\infty}\left((0, T) \times S_{\rho^{0}}^{\varepsilon}\right)}\left\|\tilde{\rho}^{\varepsilon}\right\|_{L^{\infty}\left((0, T) \times S_{\rho^{0}}^{\varepsilon}\right)}\left(\varepsilon\left\|\psi_{1}\right\|_{L^{2}\left((0, T) \times S_{\rho^{0}}^{\varepsilon}\right)}^{2}+\varepsilon^{2}\left\|\psi_{2}\right\|_{L^{2}\left((0, T) \times \tilde{S}_{\rho^{0}}^{\varepsilon}\right)}^{2}\right),
\end{aligned}
$$

and again the right-hand side tends to zero with $\varepsilon \rightarrow 0$.

Thank to Lemma 5.2 we have $\lim _{\varepsilon \rightarrow 0} \varepsilon\left\|\tilde{c}^{\varepsilon}-c\right\|_{L^{2}\left((0, T) \times \tilde{\Gamma}_{0}^{\varepsilon}\right)}^{2}=0$, and therefore

$$
\varepsilon \int_{0}^{T} \int_{\Gamma_{0}^{\varepsilon}} \lambda v\left(\tilde{\rho}^{\varepsilon}\right) \tilde{c}^{\varepsilon}\left(\psi_{1}+\varepsilon \psi_{2}\right) \mathrm{d} \gamma^{\varepsilon} \mathrm{d} t \rightarrow \int_{0}^{\tau} \int_{\Omega_{0}^{1}} \int_{\partial S} \lambda v(\rho) \tilde{c} \psi_{1} \mathrm{~d} \gamma_{y}^{\prime} \mathrm{d} x \mathrm{~d} t .
$$


Altogether, we have obtained that passing to the limit (along a subsequence) in the variational formulation (4.5) leads to the following identity

$$
\begin{aligned}
& \left.\int_{0}^{T} \int_{\Omega}\left(\chi_{\Omega_{0}^{1}}|Y|\left(v(\rho) \partial_{t} \tilde{c}+b(\rho) \partial_{w} \tilde{c}\right) \tilde{\psi}_{1}+\chi_{\Omega_{0}^{2}}\left(v(\rho) \partial_{t} \tilde{c}+b(\rho) \partial_{w} \tilde{c}\right)\right) \tilde{\psi}_{1}\right) \mathrm{d} x^{\prime} \mathrm{d} w \mathrm{~d} t \\
& \quad+\int_{0}^{T} \int_{\Omega} \int_{Y} \chi_{\Omega_{0}^{1}} \tilde{D}\left(x^{\prime}, w\right) M(\rho)\left(\nabla \tilde{c}+\nabla_{y} \tilde{c}_{1}\right) \cdot\left(\nabla \tilde{\psi}_{1}+\nabla_{y} \tilde{\psi}_{2}\right) \mathrm{d} y \mathrm{~d} x^{\prime} \mathrm{d} w \mathrm{~d} t \\
& \quad+\int_{0}^{T} \int_{\Omega} \int_{Z} \chi_{\Omega_{0}^{2}} \tilde{D}\left(x^{\prime}, w\right) M(\rho)\left(\nabla \tilde{c}+\nabla_{y} \tilde{c}_{1}\right) \cdot\left(\nabla \tilde{\psi}_{1}+\nabla_{y} \tilde{\psi}_{2}\right) \mathrm{d} y \mathrm{~d} x^{\prime} \mathrm{d} w \mathrm{~d} t \\
& =-\int_{0}^{T} \int_{\Omega} \int_{\partial S} \chi_{\Omega_{0}^{1}} \lambda v(\rho) \tilde{c} \tilde{\psi}_{1} \mathrm{~d} \gamma^{\prime} \mathrm{d} x^{\prime} \mathrm{d} w \mathrm{~d} t .
\end{aligned}
$$

Choosing $\psi_{1}=0$, we note that $c_{1}$ depends linearly in the components of $\nabla \tilde{c}$. The rest of the proof is classical in homogenization theory. Changing variables back from $w$ to $x_{3}$, the weak formulation of the equation satisfied by for $c(t, x)$, with $\tilde{c}\left(t, x^{\prime}, f\left(\rho, t, x^{\prime}, x_{3}\right)\right)=c\left(t, x^{\prime}, x_{3}\right)$ is

$$
\begin{aligned}
& \int_{0}^{T} \int_{\Omega}\left(\chi_{\Omega_{t}^{1}}|Y| \partial_{t} c+\chi_{\Omega_{t}^{2}} \partial_{t} c\right) \psi_{1} \mathrm{~d} x \mathrm{~d} t+\int_{0}^{T} \int_{\Omega} \int_{Y} \chi_{\Omega_{t}^{1}} \sum_{i, j=1}^{3} D_{i j}^{1}(x) \partial_{x_{i}} c \partial_{x_{j}} \psi_{1} \mathrm{~d} x \mathrm{~d} t \\
& +\int_{0}^{T} \int_{\Omega} \chi_{\Omega_{t}^{2}} D(x) \nabla c \cdot \nabla \psi_{1} \mathrm{~d} x \mathrm{~d} t=-\int_{0}^{T} \int_{\Omega} \int_{\partial S} \chi_{\Omega_{t}^{1}} \lambda c \psi_{1} \mathrm{~d} \gamma^{\prime} \mathrm{d} x \mathrm{~d} t,
\end{aligned}
$$

where $\Omega_{t}^{1}=\Omega \cap\left\{\left(x^{\prime}, x_{3}\right): 0<x_{3}<\rho\left(t, x^{\prime}\right)\right\}$ and $\Omega_{t}^{2}=\Omega \cap\left\{\left(x^{\prime}, x_{3}\right): \rho\left(t, x^{\prime}\right)<x_{3}<1\right\}$, and where $D_{i j}^{1}(x, t)$ is given by $D_{i 3}^{1}=D_{3 i}^{1}=D(x) \delta_{i 3}$, and for $1 \leq i \leq 2$ and $1 \leq j \leq 2$,

$$
D_{i j}^{1}(x)=D(x)\left(|Y| \delta_{i j}+\int_{Y} \partial_{y_{i}} \omega_{j} \mathrm{~d} y\right),
$$

where $\omega_{j}$ are $Z$ periodic solutions of

$$
-\Delta_{y} \omega_{j}=0 \text { in } Y, \quad-\nabla_{y}\left(w_{j}-y_{j}\right) \cdot \nu=0 \text { on } \partial S .
$$

they are uniquely defined if a normalisation condition is chosen. Because of the symmetries of the problem, $\int_{Y} \partial_{y_{1}} \omega_{1} \mathrm{~d} y=\int_{Y} \partial_{y_{2}} \omega_{2} \mathrm{~d} y$, and $\int_{Y} \partial_{y_{2}} \omega_{1} \mathrm{~d} y=\int_{Y} \partial_{y_{1}} \omega_{2} \mathrm{~d} y=0$. We have obtained that $D^{1}$ is diagonal, and given by $D_{33}^{1}=D(x)$ and $D_{11}^{1}=D_{11}^{1}=D(x)\left(|Y|+d^{*}\right)$, as announced.

Finally, the convergence of initial data follows from the strong convergence of $\rho^{\varepsilon}$, the two-scale convergence of $c^{\varepsilon}$ and of $\partial_{t} c^{\varepsilon}$ and from the identity

$$
\begin{aligned}
& \int_{0}^{T} \int_{\Omega_{0}^{\varepsilon}} v\left(\rho^{\varepsilon}\right) \partial_{t} c^{\varepsilon} \psi\left(x, \frac{x}{\varepsilon}\right) \xi(t) \mathrm{d} x^{\prime} \mathrm{d} t=-\int_{\Omega_{0}^{\varepsilon}} v\left(\rho_{0}^{\varepsilon}\right) c^{0} \psi\left(x, \frac{x}{\varepsilon}\right) \xi(0) \mathrm{d} x^{\prime} \\
& -\int_{0}^{T} \int_{\Omega_{0}^{\varepsilon}} c^{\varepsilon} \psi\left(x, \frac{x}{\varepsilon}\right)\left(\partial_{t} v\left(\rho^{\varepsilon}\right) \xi(t)+v\left(\rho^{\varepsilon}\right) \partial_{t} \xi(t)\right) \mathrm{d} x^{\prime} \mathrm{d} t,
\end{aligned}
$$

with $\xi \in C^{\infty}([0, T]), \xi(T)=0$ and $\psi \in C^{\infty}(\Omega \times Z)$. 
This conclude the proof of convergence, up to sub-sequences. We shall now show the uniqueness of the solution of the limit problem, which will imply the convergence of the entire sequence of microscopic problems.

\section{Uniqueness.}

The uniqueness of solution to the macroscopic problem (3.1)-(3.3) follows from a variant of the proof of uniqueness for the $\varepsilon$ dependent problem. Integrating the equation defining $\rho$ implicitly, we obtain

$$
\rho\left(t, x^{\prime}\right)=K-\left(K-\rho^{0}\left(x^{\prime}\right)\right) \exp \left(-\int_{0}^{t} \frac{c s\left(s, x^{\prime}\right)}{\sqrt{1+c s^{2}\left(s, x^{\prime}\right)}} \mathrm{d} s\right) .
$$

Following the same steps that in Proposition 4.4 and Lemma 4.5, we verify the maximum principle holds for $c$, and that

$$
\left|\nabla_{x^{\prime}} \rho\right| \leq \mu\left(\left\|\rho^{0}\right\|_{C^{1}\left([0,1]^{2}\right)}+\max _{\bar{\Omega}} c^{0}\right) .
$$

Then, given any two solutions $\left(\rho_{a}, \tilde{c}_{a}\right)$ and $\left(\rho_{b}, \tilde{c}_{b}\right)$, where the ${ }^{\sim}$ corresponds to the change of domain from $\Omega_{t}$ to $\Omega_{0}$ we obtain, as in Proposition 4.9 that

$$
\sup _{(0,1)^{2} \times(0, T)}\left|\rho_{a}-\rho_{b}\right|+\sup _{(0,1)^{2} \times(0, T)}\left|\nabla_{x^{\prime}} \rho_{a}-\nabla_{x^{\prime}} \rho_{b}\right| \leq \mu\left\|\tilde{c}_{a}-\tilde{c}_{b}\right\|_{L^{1}\left(\Omega_{0} \times(0, T)\right)}
$$

Repeating then the computation done Proposition 4.9, we obtain for any $\tau<T$,

$$
\int_{\Omega_{0}}\left|\tilde{c}_{a}(\tau)-\tilde{c}_{b}(\tau)\right|^{2} \mathrm{~d} x^{\prime} \mathrm{d} w \leq \mu \int_{0}^{\tau} \int_{\Omega_{0}}\left|\tilde{c}_{a}-\tilde{c}_{b}\right|^{2} \mathrm{~d} x^{\prime} \mathrm{d} w \mathrm{~d} t
$$

and the conclusion follows from Gronwall's Lemma.

\section{CONCLUding REMARKS}

Using homogenization techniques we derived a macroscopic model for nutrient diffusion and plant root growth from a microscopic description on the scale of a single root. We have shown the convergence of the sequence of solutions of full problem involving interactions at the microscopic scale to a solution of a macroscopic problem defined in the homogeneous domain $\Omega$. The influence of the micro-structure problem appears in the diffusion coefficient and in the absorption term in the macroscopic model in a suitably averaged form. An essential feature of the microscopic problem is the non-local nature of the root growth rate. This feature is preserved in the limit. We view this model as a first step towards a more precise modelling of the dependence of root growth on the nutrient concentration. To clarify the presentation, we restricted ourselves to a very simple root geometry. One improvement of this model could be to make the growth rate of the roots depend on the quantity of nutrient available very locally around the root. This would mean, for example, to allow $r_{0}$, the radius of the ball centred around each root tip which determines each root growth rate, to depend on $\varepsilon$. Another variant would be to allow the velocity to depend upon for point-wise values of the nutrient concentration at the tip of the roots. We do not know however if such assumptions would lead to well-posed models.

A second natural generalization would be to allow roots to growth directions to depend on other factors than gravity, leading to complex root networks. This will be the subject of future studies.

Acknowledgements. The authors would like to thank the reviewers for their suggestions and comments which significantly improved the presentation of this paper. Yves Capdeboscq is supported by the EPSRC Science and Innovation award to the Oxford Centre for Nonlinear PDE (EP/E035027/1). This work was completed while Yves Capdeboscq was visiting the Institute for Advanced Study, and the author would like to thank the School of Mathematics for the wonderful time he had there. This material is based upon work supported by the National Science Foundation under agreement 
No. DMS-0635607. Any opinions, findings and conclusions or recommendations expressed in this material are those of the authors and do not necessarily reflect the views of the National Science Foundation.

\section{REFERENCES}

[1] E. Acerbi, V. Chiado Piat, G. Dal Maso and D. Percivale, An extension theorem from connected sets, and homogenization in general periodic domains. Nonlinear Anal. 18 (1992) 481-496.

[2] G. Allaire, Homogenization and two-scale convergence. SIAM J. Math. Anal. 23 (1992) 1482-1518.

[3] G. Allaire, A. Damlamian and U. Hornung, Two-scale convergence on periodic surfaces and applications, in Proceedings of the International Conference on Mathematical Modelling of Flow through Porous Media, edited by A. Bourgeat et al., World Scientific Pub., Singapore (1996) 15-25.

[4] P. Bastian, A. Chavarría-Krauser, Ch. Engwer, W. Jäger, S. Marnach and M. Ptashnyk, Modelling in vitro growth of dense root networks. J. Theor. Biol. 254 (2008) 99-109.

[5] M. Caloin and O. Yu, An extension of the logistic model of plant growth. Ann. Bot. 49 (1982) 599-607.

[6] A. Chavarria-Krauser and U. Schurr, A cellular growth model for root tips. J. Theor. Biol. 230 (2004) 21-32.

[7] S. Chuai-Aree, W. Jäger, H.G. Bock and S. Siripant, Modeling, simulation and visualization of plant root growth and diffusion processes in soil volume, 4th International Workshop on Functional-Structural Plant Models, edited by C. Godin et al. Montpellier, France (2004) 289-293.

[8] D. Cioranescu and J. Saint Jean Paulin, Homogenization of reticulated structures. Springer (1999).

[9] L. Dupuy, T. Fourcaud, A. Stokes and F. Danjon, A density-based approach for the modelling of root architecture: application to Maritime pine (Pinus pinaster Ait.) root systems. J. Theor Biol. 236 (2005) 323-334.

[10] L. Dupuy, P.J. Gregory and A.G. Bengough, Root growth models: towards a new generation of continuous approaches. J. Exp. Bot. 61 (2010) 2131-2143.

[11] R.O. Erickson, Modeling of plant growth. Ann. Rev. Plant Physiol. 27 (1976) 407-434.

[12] P. Grabarnik, L. Pagès and A.G. Bengough, Geometrical properties of simulated maize root systems: consequences for length density and intersection density. Plant Soil 200 (1998) 157-167.

[13] A. Marciniak-Czochra and M. Ptashnyk, Derivation of a macroscopic receptor-based model using homogenization techniques, SIAM J. Math. Anal. 40 (2008) 215-237.

[14] G. Nguetseng, A general convergence result for a functional related to the theory of homogenization. SIAM J. Math. Anal. 20 (1989) 608-623.

[15] L. Pagès, How to include organ interactions in models of the root system architecture? The concept of endogenous environment. Ann. For. Sci. 57 (2000) 535-541.

[16] L. Pagès, M.O. Jordan and D. Picard, A simulation-model of the three-dimensional architecture of the maize root-system. Plant Soil 1989 (1989) 147-154.

[17] L. Pagès, G. Vercambre, J.-L. Drouet, F. Lecompte, C. Collet and J. Le Bot, Root Typ: a generic model to depict and analyse the root system architecture. Plant Soil 258 (2004) 103-119.

[18] P. Prusinkiewicz, Modeling plant growth and development. Current Opinion in Plant Biol. 7 (2004) 79-83.

[19] P. Prusinkiewicz and A. Lindenmayer, The algorithmic beauty of plants. Springer-Vergal, New York, USA (1990).

[20] O. Wilderotter, An adaptive numerical method for the Richards equation with root growth. Plant Soil 251 (2003) 255-267. 